\title{
FROM NOMINAL TO HIGHER-ORDER REWRITING AND BACK AGAIN
}

\author{
JESÚS DOMÍNGUEZ AND MARIBEL FERNÁNDEZ
}

Department of Informatics, King's College London, Strand WC2R 2LS, UK

e-mail address: \{jesus.dominguez_alvarez, maribel.fernandez\}@kcl.ac.uk

\begin{abstract}
We present a translation function from nominal rewriting systems (NRSs) to combinatory reduction systems (CRSs), transforming closed nominal rules and ground nominal terms to CRSs rules and terms, respectively, while preserving the rewriting relation. We also provide a reduction-preserving translation in the other direction, from CRSs to NRSs, improving over a previously defined translation. These tools, together with existing translations between CRSs and other higher-order rewriting formalisms, open up the path for a transfer of results between higher-order and nominal rewriting. In particular, techniques and properties of the rewriting relation, such as termination, can be exported from one formalism to the other.
\end{abstract}

\section{INTRODUCTION}

Programs and logical systems often include binding operators. Term rewriting systems [1, 4, in their standard form, do not provide support for reasoning on binding structures. This motivated the study of combinations of first-order rewriting systems with the $\lambda$-calculus [2], which offers a notion of variable binding and substitution. Combinatory reduction systems (CRSs) [24, 25] are well-known examples of higher-order rewriting formalisms, where a meta-language based on the untyped $\lambda$-calculus was incorporated to a first-order rewriting framework. Other approaches followed, such as HRSs [29] and ERSs [23, 20] for example.

Techniques to prove confluence and termination of higher-order rewriting systems were studied in [28, 25, 21] amongst others. However, the syntax and type restrictions imposed on rules in these systems have prevented the design of completion procedures for higher-order rewriting systems [30].

More recently, the nominal approach [19, 31] has been used to design rewriting systems with support for binding [14]. Nominal rewriting systems do not rely on the $\lambda$-calculus, instead, two kinds of variables are used: atoms, which can be abstracted but behave similarly to constants thus allowing explicit manipulation, and meta-level variables or just variables, which are first-order in that they cannot be abstracted and substitution does not avoid capture of unabstracted atoms. On nominal terms [34, 35, 14 $\alpha$-equivalence is axiomatised using bijective mappings on atoms, known as permutations, and a freshness relation between

2012 ACM CCS: [Theory of computation]: Logic - Equational logic and rewriting.

Key words and phrases: rewriting, nominal syntax, Combinatory Reduction Systems, higher-order syntax, translation tool. 
atoms and terms. Nominal syntax enjoys many useful properties, for instance, unification modulo $\alpha$-equivalence is decidable and unitary [34, 35] and nominal matching is linear [5]. Nominal rewriting can be implemented efficiently if rules are closed (roughly speaking, closed rules do not contain free atoms, and preserve the free/abstracted status of atoms during reduction - a natural restriction, which is also imposed on CRSs, HRSs and ERSs by definition).

The availability of efficient algorithms to solve unification problems on nominal terms motivated the study of the relationship between higher-order and nominal syntax in a series of papers [6, 16, 27]. In this paper, we focus on the relationship between higher-order and nominal rewriting, specifically between CRSs and NRSs. The translations provided in [6, 27] preserve the unifiability relation, whereas ours preserves the rewriting relation, which is key to the translation of properties such as confluence and termination. We define a translation function from closed NRS rules and ground nominal terms to CRS rules and terms, preserving the rewriting relation. Then, we give a translation function from CRSs to NRSs, improving over a previous translation described in [16. Since we now have reduction-preserving translations in both directions, properties and techniques developed for one formalism can be exported to the other (e.g., termination techniques based on the construction of a well-founded reduction ordering). A Haskell implementation of the translation functions, along with a tool to prove termination using the nominal recursive path ordering [17, are available from [10, 9].

Related work. CRSs, HRSs and ERSs are well-known examples of higher-order rewriting formalisms. A comparison of various higher-order rewriting formalisms, with many interesting examples, is provided in [37; see also [22] for a concise presentation of higher-order rewrite systems. In [36, 20], CRSs are compared with HRSs and ERSs respectively, and in [3] CRSs are expressed in terms of the $\rho$-calculus [7, 8]. In [26, a termination-preserving translation between Algebraic Functional Systems and other higher-order formalisms is presented. Although in this paper we focus on the relationship between NRSs and CRSs, thanks to the existing translations between CRSs and other higher-order rewriting formalisms, this is sufficient to obtain a bridge between nominal and higher-order rewriting.

Our work is closely related to the work reported in [6, 27]: Cheney [6] represented higherorder unification as nominal unification, and Levy and Villaret [27] transformed nominal unification into higher-order unification, providing a translation that preserves unifiers. Our translation differs from [6, 27] in that our requirement is to have a mapping of NRS ground terms and rules to CRS terms and rules in such a way that reductions are preserved.

This paper is an updated and extended version of [11. We have included here, in addition to the translation from NRSs to CRSs given in [11, all the proofs previously omitted due to space constraints as well as a translation from CRSs to NRSs, improving on a previous result given in [16. We provide detailed explanations, and illustrate the translations with examples.

Overview of the paper. The rest of the paper is organised as follows. In section 2 we recall both formalisms, CRSs and NRSs, as defined in [24] and [14 respectively. In section 3 we describe in detail the translation of nominal terms to CRS meta-terms, while in section 4 we extend it to take into account rules and substitution. In Section 5 we prove that nominal rewrite steps can be simulated in CRSs via the translation function. Section 6 presents 
a translation from CRSs to NRSs. In section 7 we show examples of application of the translations. Section 8 concludes and discusses future work.

\section{Preliminaries}

We start by briefly recalling the main concepts of nominal rewrite systems and combinatory reduction systems - two rewriting formalisms that extend the syntax of first-order terms and the notion of rewriting, to facilitate the specification of systems with binding operators. We refer the reader to [25, 14] for more details and examples.

2.1. Nominal Rewriting. A nominal signature $\Sigma$ is a set of term-formers, or function symbols, $f, g, \ldots$, each with a fixed arity. Fix a countably infinite set $\mathcal{X}$ of variables ranged over by $X, Y, Z, \ldots$, and a countably infinite set $\mathcal{A}$ of atoms ranged over by $a, b, c, \ldots$, and assume that $\Sigma, \mathcal{X}$ and $\mathcal{A}$ are pairwise disjoint. We follow the permutative convention [18, Convention 2.3] where $a, b, c, \ldots$ range over distinct atoms unless stated otherwise.

Permutations $\pi$ are bijections on $\mathcal{A}$ such that the set of atoms for which $a \neq \pi(a)$ is finite; this is called the support of $\pi$, written as support $(\pi)$. A swapping is a pair of atoms, written $(a b)$. Permutations are represented by lists of swappings, Id denotes the identity permutation. We write $\pi^{-1}$ for the inverse of $\pi$ and $\pi \circ \pi^{\prime}$ for the composition of $\pi^{\prime}$ and $\pi$. For example, if $\pi=(a b)(b c)$, then $\pi(a)=b, \pi^{-1}=(b c)(a b)$ and $\pi^{-1}(a)=c$.

Definition 2.1 (Syntax). Nominal terms, or just terms if there is no ambiguity, are generated by the grammar

$$
s, t::=a|\pi \cdot X|[a] s|f s|\left(s_{1}, \ldots, s_{n}\right)
$$

and called, respectively, atoms, moderated variables or simply variables, abstractions, function applications (which must respect the arity of the function symbol) and tuples; if the arity of $f$ is 0 we may omit the parentheses in the application. We abbreviate $\mathrm{Id} \cdot X$ as $X$ if there is no ambiguity. An abstraction $[a] t$ is intended to represent $t$ with $a$ bound; we say that the scope of $[a]$ is $t$. Call occurrences of a abstracted if they are in the scope of an abstraction, and unabstracted (or free) otherwise.

For example, $f(X,(a b) \cdot X)$ is a nominal term, and so is $f([a] X,[b] b)$. The latter term has $X$ in the scope of $[a]$ and $b$ in the scope of $[b]$. For more examples, we refer the reader to [34, 35, 14].

Definition 2.2. The functions $V(t)$ and $A(t)$ are used to compute the sets of variables and atoms in a nominal term $t$, respectively. They are inductively defined as follows:

$$
\begin{gathered}
V(a)=\varnothing \quad V([a] t)=V(t) \quad V(\pi \cdot X)=\{X\} \\
V(f s)=V(s) \quad V\left(\left(s_{1}, \ldots, s_{n}\right)\right)=V\left(s_{1}\right) \cup \ldots \cup V\left(s_{n}\right) \\
A(a)=\{a\} \quad A([a] t)=A(t) \cup\{a\} \quad A(\pi \cdot X)=\operatorname{support}(\pi) \\
A(f s)=A(s) \quad A\left(\left(s_{1}, \ldots, s_{n}\right)\right)=A\left(s_{1}\right) \cup \ldots \cup A\left(s_{n}\right)
\end{gathered}
$$

Ground terms have no variables: $V(t)=\varnothing$.

Notice that $V(t)$ is a syntactic notion, whereas $A(t)$ takes into account the semantics of permutations (represented as lists of swappings). For example, $A(f(a,[b](c d)(e f)(f e) \cdot X))$ $=\{a, b, c, d\}$. 
Definition 2.3 (Positions and subterms of nominal terms). Let $s$ be a nominal term. The set $\mathcal{P} o s(s)$ of positions in $s$ is a set of strings of positive integers, inductively defined below. We also define below the subterms of $s:\left.s\right|_{p}$ denotes the subterm of $s$ at position $p$.

- if $s=a$ or $s=\pi \cdot X$, then $\mathcal{P} o s(s)=\{\epsilon\}$ and $\left.s\right|_{\epsilon}=s$, where $\epsilon$ denotes the empty string;

- if $s=[a] t$, then $\operatorname{Pos}(s)=\{\epsilon\} \cup\{1 \cdot p \mid p \in \mathcal{P}$ os $(t)\},\left.s\right|_{\epsilon}=s$ and $\left.s\right|_{1 \cdot p}=\left.t\right|_{p}$;

- if $s=f t$, then $\operatorname{Pos}(s)=\{\epsilon\} \cup\{1 \cdot p \mid p \in \mathcal{P} o s(t)\},\left.s\right|_{\epsilon}=s$ and $\left.s\right|_{1 \cdot p}=\left.t\right|_{p}$;

- if $s=\left(t_{1}, \ldots, t_{n}\right)$, then $\mathcal{P} o s(s)=\{\epsilon\} \cup \bigcup_{i=1}^{n}\left\{i \cdot p \mid p \in \mathcal{P}_{o s}\left(t_{i}\right)\right\},\left.s\right|_{\epsilon}=s$ and $\left.s\right|_{i \cdot p}=\left.t_{i}\right|_{p}$.

The position $\epsilon$ is called the root position of the term $s$, and the symbol at this position is called the root symbol of $s$.

We now extend the action of permutations to terms. Recall we use the permutative convention, so atoms $a, b, c$ are considered distinct among them.

Definition 2.4 (Permutation action). The action of a permutation $\pi$ on a term $t$, written $\pi \cdot t$, is defined by induction: Id $t=t$ and $(a b) \pi \cdot t=(a b) \cdot(\pi \cdot t)$, where a swapping acts inductively on the structure of terms as follows:

$$
\begin{aligned}
& (a b) \cdot a=b \quad(a b) \cdot b=a \quad(a b) \cdot c=c \\
& (a b) \cdot(\pi \cdot X)=((a b) \circ \pi) \cdot X \quad(a b) \cdot[c] t=[c](a b) \cdot t \\
& (a b) \cdot[a] t=[b](a b) \cdot t \quad(a b) \cdot[b] t=[a](a b) \cdot t \\
& (a b) \cdot f t=f(a b) \cdot t \quad(a b) \cdot\left(t_{1}, \ldots, t_{n}\right)=\left((a b) \cdot t_{1}, \ldots,(a b) \cdot t_{n}\right) .
\end{aligned}
$$

Substitutions map variables to terms, and act on terms without avoiding capture of atoms, according to the following definition.

Definition 2.5 (Substitution). Substitutions are generated by the grammar:

$$
\sigma::=\operatorname{Id} \mid[X \mapsto s] \sigma
$$

We use the same notation for the identity substitution and permutation, and also for composition, since there will be no ambiguity.

Write $t \sigma$ for the application of $\sigma$ on $t$, defined as follows:

$$
\begin{gathered}
t \operatorname{Id}=t \quad t[X \mapsto s] \sigma=(t[X \mapsto s]) \sigma \quad \text { where } \\
a[X \mapsto s]=a \quad(\pi \cdot X)[X \mapsto s]=\pi \cdot s \quad(\pi \cdot Y)[X \mapsto s]=\pi \cdot Y \quad(X \neq Y) \\
([a] t)[X \mapsto s]=[a](t[X \mapsto s]) \quad(f t)[X \mapsto s]=f t[X \mapsto s] \\
\left(t_{1}, \ldots, t_{n}\right)[X \mapsto s]=\left(t_{1}[X \mapsto s], \ldots, t_{n}[X \mapsto s]\right) .
\end{gathered}
$$

The domain of a substitution $\sigma, \operatorname{dom}(\sigma)$, is the set of variables such that $X \sigma \neq X$. The restriction of a substitution $\sigma$ to a set of variables $V$, written $\left.\sigma\right|_{V}$, is defined as $\left.\sigma\right|_{V}=[X \rightarrow$ $X \sigma \mid X \in V]$.

The semantics of nominal terms is defined using nominal sets [32]. A $\operatorname{Perm}(\mathcal{A})$-set is a set $T$ equipped with a permutation action, such that $\operatorname{Id} \cdot x=x$ and $\pi \cdot\left(\pi^{\prime} \cdot x\right)=\left(\pi \circ \pi^{\prime}\right) \cdot x$ for each object $x \in T$. A set $S$ of atoms supports $x \in T$ if for all atoms $a, b \notin S,(a b) \cdot x=x$. A nominal set is a $\operatorname{Perm}(\mathcal{A})$-set where each element has finite support. Nominal terms form a nominal set, using $\alpha$-equivalence as equality [32]. To define the support of a term, we introduce the notion of freshness. The support set of a term $t$, abbreviated $\operatorname{supp}(t)$, is the 
complement of the set of fresh atoms in $t$. When a term $t$ is ground, $\operatorname{supp}(t)$ coincides with the syntactic notion of unabstracted atoms in $t$.

Definition 2.6 (Freshness). A freshness constraint is a pair a\#t of an atom and a term. A freshness context (ranged over by $\Delta, \nabla, \Gamma$ ), is a set of constraints of the form $a \# X$. Freshness judgements, written $\Delta \vdash a \# t$, are derived using the rules below.

$$
\begin{aligned}
& \overline{\Delta \vdash a \# b}(\# \mathbf{a b}) \frac{\pi^{-1} \cdot a \# X \in \Delta}{\Delta \vdash a \# \pi \cdot X}(\# \mathbf{X}) \frac{\Delta \vdash a \# s}{\Delta \vdash a \# f s}(\# \mathbf{f}) \\
& \frac{\Delta \vdash a \# s_{1} \cdots \Delta \vdash a \# s_{n}}{\Delta \vdash a \#\left(s_{1}, \ldots, s_{n}\right)}(\# \text { tupl }) \quad \frac{\Delta \vdash a \# s}{\Delta \vdash a \#[a] s}(\#[\mathbf{a}]) \frac{\Delta}{\Delta \vdash a \#[b] s}(\#[\mathbf{b}])
\end{aligned}
$$

For example, $a \# X \vdash b \#(a b) \cdot X$ can be derived using rule $(\# \mathbf{X})$, since $(a b) \cdot a=b$.

In nominal languages, one is interested in terms $t$ that have finite support, because for them there exists always a fresh atom $a$ such that $a \# t$ (recall the set $\mathcal{A}$ of atoms is infinite).

Definition 2.7 ( $\alpha$-equivalence). An $\alpha$-equality constraint is a pair $s \approx_{\alpha} t$ of terms. Equivalence judgements, written $\Delta \vdash s \approx_{\alpha} t$, are derived using the rules below, where $d s\left(\pi, \pi^{\prime}\right)=$ $\left\{a \in \mathcal{A} \mid \pi \cdot a \neq \pi^{\prime} \cdot a\right\}$ (difference set).

$$
\begin{gathered}
\frac{\forall a \in d s\left(\pi, \pi^{\prime}\right): a \# X \in \Delta}{\Delta \vdash a \approx_{\alpha} a}(\approx \alpha \mathbf{a}) \quad \frac{\Delta \vdash s \approx_{\alpha} t}{\Delta \vdash \pi \cdot X \approx_{\alpha} \pi^{\prime} \cdot X}\left(\approx_{\alpha} \mathbf{X}\right) \quad \frac{\Delta \vdash f s \approx_{\alpha} f t}{\left.\Delta \approx_{\alpha} \mathbf{f}\right)} \\
\frac{\Delta \vdash s_{1} \approx_{\alpha} t_{1} \cdots \Delta \vdash s_{n} \approx_{\alpha} t_{n}}{\Delta \vdash\left(s_{1}, \ldots, s_{n}\right) \approx_{\alpha}\left(t_{1}, \ldots, t_{n}\right)}\left(\approx_{\alpha} \text { tupl }\right) \\
\frac{\Delta \vdash s \approx_{\alpha} t}{\Delta \vdash[a] s \approx_{\alpha}[a] t}\left(\approx_{\alpha}[\mathbf{a}]\right) \quad \frac{\Delta \vdash(b a) \cdot s \approx_{\alpha} t \quad \Delta \vdash b \# s}{\Delta \vdash[a] s \approx_{\alpha}[b] t}\left(\approx_{\alpha}[\mathbf{b}]\right)
\end{gathered}
$$

Let $P_{i}$ be a freshness or $\alpha$-equality constraint (for $1 \leq i \leq n$ ). We write $\Delta \vdash P_{1}, \ldots, P_{n}$ when proofs of $\Delta \vdash P_{i}$ exist (for $1 \leq i \leq n$ ), using the derivation rules above.

The relation $\approx_{\alpha}$ is indeed an equivalence relation [34, 35].

Example 2.8. We can derive $a \# X \vdash[a](a b) \cdot X \approx_{\alpha}[b] X$ as follows.

$$
\frac{\frac{d s((b a)(a b), \mathrm{Id})=\varnothing}{a \# X \vdash(b a)(a b) \cdot X \approx_{\alpha} X}\left(\approx_{\alpha} \mathbf{X}\right) \quad \frac{}{a \# X \vdash b \#(a b) \cdot X}(\# \mathbf{X})}{a \# X \vdash[a](a b) \cdot X \approx_{\alpha}[b] X}\left(\approx_{\alpha}[\mathbf{b}]\right)
$$

Property 2.9 (14, Lemma 23). For any $a \in \mathcal{A}$, if $\Delta \vdash a \# s$ and $\Delta \vdash s \approx_{\alpha} t$ then $\Delta \vdash a \# t$. Hence, if $\Delta \vdash s \approx_{\alpha} t$ then $s$ and $t$ have the same support set.

Definition 2.10 (Nominal rewrite system). A nominal rewrite rule $R=\nabla \vdash l \rightarrow r$ is a tuple of a freshness context $\nabla$ and terms $l$ and $r$ such that $V(r) \cup V(\nabla) \subseteq V(l)$.

A nominal rewrite system (NRS) is an equivariant set $\mathcal{R}$ of nominal rewrite rules, that is, a set of nominal rules that is closed under permutations. We shall generally equate a set of rewrite rules with its equivariant closure.

Definition 2.11. We extend the notions given in Definition 2.2 for both variables, $V(t)$, and atoms, $A(t)$, to include rules, contexts, substitutions, etc. Particularly, for contexts $A(\Delta)=\{a \mid a \# X \in \Delta$ for some $X\}$ and for substitutions, $A(\sigma)=\{A(X \sigma) \mid X \in \operatorname{dom}(\sigma)\}$. 
Example 2.12. The following rules are used to compute prenex normal forms in first-order logic. The signature has term-formers forall, exists, not, and. Intuitively, equivariance means that the choice of atoms in rules is not important (see [14 for more details), therefore we could change below $a$ to $b$, for instance.

$$
\begin{array}{lll}
a \# P & \vdash \text { and }(P, \text { forall }([a] Q)) & \rightarrow \text { forall }([a] \operatorname{and}(P, Q)) \\
a \# P & \vdash \text { and }(\text { forall }([a] Q), P) & \rightarrow \text { forall }([a] \operatorname{and}(Q, P)) \\
a \# P & \vdash \text { or }(P, \text { forall }([a] Q)) & \rightarrow \text { forall }([a] \operatorname{or}(P, Q)) \\
a \# P & \vdash \operatorname{or}(\text { forall }([a] Q), P) & \rightarrow \text { forall }([a] \operatorname{or}(Q, P)) \\
a \# P & \vdash \text { and }(P, \operatorname{exists}([a] Q)) & \rightarrow \text { exists }([a] \operatorname{and}(P, Q)) \\
a \# P & \vdash \operatorname{and}(\operatorname{exists}([a] Q), P) & \rightarrow \operatorname{exists}([a] \operatorname{and}(Q, P)) \\
a \# P & \vdash \operatorname{or}(P, \operatorname{exists}([a] Q) & \rightarrow \operatorname{exists}([a] \operatorname{or}(P, Q)) \\
a \# P & \vdash \operatorname{or}(\operatorname{exists}([a] Q), P) & \rightarrow \operatorname{exists}[a] \operatorname{or}(Q, P) \\
& \vdash \operatorname{not}(\operatorname{exists}([a] Q)) & \rightarrow \text { forall }([a] \operatorname{not}(Q)) \\
& \vdash \operatorname{not}(\text { forall }([a] Q)) & \rightarrow \operatorname{exists}([a] \operatorname{not}(Q)) .
\end{array}
$$

Nominal rewriting [14] operates on terms-in-contexts, written $\Delta \vdash s$ or just $s$ if $\Delta=\varnothing$. Below, $C[$ ] varies over terms with exactly one occurrence of a distinguished variable Id--, or just -. We write $C[s]$ for $C[-\mapsto s]$, and $\Delta \vdash \nabla \theta$ for $\{\Delta \vdash a \# X \theta \mid a \# X \in \nabla\}$.

Definition 2.13 (Nominal rewriting). A term $s$ rewrites with $R=\nabla \vdash l \rightarrow r$ to $t$ in $\Delta$, written $\Delta \vdash s \rightarrow_{R} t$ (as usual, we assume $V(R) \cap(V(\Delta) \cup V(s))=\varnothing$ ), if $s=C\left[s^{\prime}\right]$ and there exists $\theta$ such that $\Delta \vdash \nabla \theta, \Delta \vdash l \theta \approx_{\alpha} s^{\prime}$, and $\Delta \vdash C[r \theta] \approx_{\alpha} t$. Since $\Delta$ does not change during rewriting, a rewriting derivation is written $\Delta \vdash s_{1} \rightarrow_{R} s_{2} \rightarrow_{R} \ldots \rightarrow_{R} s_{n}$, abbreviated as $\Delta \vdash s_{1} \rightarrow^{*} s_{n}$.

When rules are closed, nominal rewriting can be efficiently implemented using nominal matching (then, there is no need to consider equivariance). We define closed rewriting below, after defining closed terms.

Closed terms are, roughly speaking, terms without unabstracted atoms, such that variables behave uniformly with respect to their support. We give a definition below.

Definition 2.14 (Closedness). A term-in-context $\Delta \vdash t$ is closed if it satisfies the following conditions:

(1) if $\left.t\right|_{p}=a$ then $\left.t\right|_{p}$ is in the scope of an abstraction for $a$;

(2) if $\pi \cdot X$ occurs in the scope of an abstraction of $\pi \cdot a$ then any occurrence of $\pi^{\prime} \cdot X$ occurs in the scope of an abstraction of $\pi^{\prime} \cdot a$ or $a \# X \in \Delta$;

(3) for any pair $\pi_{1} \cdot X, \pi_{2} \cdot X$ occurring in $t$, and $a \in d s\left(\pi_{1}, \pi_{2}\right)$, if $a$ is not abstracted in one of the occurrences then $a \# X \in \Delta$.

A rewrite rule $\nabla \vdash l \rightarrow r$ is closed if $\nabla \vdash(l, r)$ is a closed term.

The first condition in the definition specifies that no atom occurs unabstracted in a closed term. The second condition states that if an atom $a$ in an instance of a variable $\pi \cdot X$ is captured (i.e. $\pi \cdot X$ is under an abstraction for $\pi \cdot a$ ) then it is captured in all occurrences of $X$, otherwise it is fresh for $X$. The third condition says that if two occurrences of $X$ have different suspended permutations, then any atom in the difference set that could occur in an instance of $X$ is captured.

For example, $[a] f(X, a)$ is closed, but $f(X, a)$ and $f(X,[a] X)$ are not, however $a \# X \vdash$ $f(X,[a] X)$ is closed. All the rewrite rules in Example 2.12 are closed. 
Closedness can be easily checked using the nominal matching algorithm [5] as follows. First, given a term in context $\nabla \vdash t$, or more generally, a pair $P=\nabla \vdash(l, r)$ (this could be a rule $R=\nabla \vdash l \rightarrow r)$, let us write $P^{n}=\nabla^{n} \vdash\left(l^{n}, r^{n}\right)$ to denote a freshened variant of $P$, i.e., a version where the atoms and variables have been replaced by 'fresh' ones. We shall always explicitly say what $P^{n}$ is freshened for when this is not obvious. For example, a freshened version of $(a \# X \vdash f(X) \rightarrow X)$ with respect to itself and to $a^{\prime} \# X \vdash a^{\prime}$ is $\left(a^{\prime \prime} \# X^{\prime} \vdash f\left(X^{\prime}\right) \rightarrow X^{\prime}\right)$. We will write $A\left(P^{\prime}\right) \# V(P)$ to mean that all atoms in $P^{\prime}$ are fresh for each of the variables occurring in $P$. Let $\nabla^{n} \vdash t^{n}$ be a freshened version of $\nabla \vdash t$. Then $\nabla \vdash t$ is closed if there exists a substitution $\sigma$ such that $\nabla, A\left(\nabla^{n} \vdash t^{n}\right) \# V(\nabla \vdash t) \vdash \nabla^{n} \sigma$ and $\nabla, A\left(\nabla^{n} \vdash t^{n}\right) \# V(\nabla \vdash t) \vdash t^{n} \sigma \approx_{\alpha} t$. A similar check can be done for nominal rewrite rules.

Definition 2.15 (Closed rewriting). Let $R^{n}$ be a freshened version of the rule $R$ with respect to $\Delta, s, t$ (i.e., a version where the atoms and variables in $R$ have been replaced by fresh ones; as shown in [14, it does not matter which particular freshened $R^{n}$ we choose). We write $\Delta \vdash s \rightarrow_{R}^{c} t$ if $\Delta, \Delta^{\prime} \vdash s \rightarrow_{R^{n}} t$, where $\Delta^{\prime}=A\left(R^{n}\right) \# V(\Delta, s)$, and call this a closed rewriting step. The subindex $R$ may be omitted if it is clear from the context.

Closed NRSs inherit properties of first-order rewriting systems such as the Critical Pair Lemma [14.

Example 2.16. We show a closed rewriting step for the term $\vdash$ and $(X$, forall $([b] \mathbf{f}(b)))$ using the first rule in Example 2.12.

$$
\vdash \text { and }(X, \text { forall }([b] \mathrm{f}(b))) \rightarrow \rightarrow^{c} \text { forall }\left(\left[a^{\prime}\right] \text { and }\left(X, \mathrm{f}\left(a^{\prime}\right)\right)\right)
$$

To generate it, we first obtain a freshened variant of the rule with respect to itself and the given term: $a^{\prime} \# P^{\prime} \vdash$ and $\left(P^{\prime}\right.$, forall $\left.\left(\left[a^{\prime}\right] Q^{\prime}\right)\right) \rightarrow$ forall $\left(\left[a^{\prime}\right]\right.$ and $\left.\left(P^{\prime}, Q^{\prime}\right)\right)$. Notice that there is a rewrite step

$$
a^{\prime} \# X \vdash \text { and }(X, \text { forall }([b] \mathrm{f}(b))) \rightarrow \text { forall }\left(\left[a^{\prime}\right] \operatorname{and}\left(X, \mathrm{f}\left(a^{\prime}\right)\right)\right) .
$$

using the matching substitution $\theta=\left[P^{\prime} \mapsto X\right]\left[Q^{\prime} \mapsto f\left(a^{\prime}\right)\right]$, since $a^{\prime} \# P^{\prime} \theta$ holds.

2.2. Combinatory Reduction Systems. A combinatory reduction system (CRS) 24, 25] is a pair consisting of an alphabet $\mathbb{A}$ and a set of rewrite rules.

The CRS alphabet $\mathbb{A}$ consists of:

(1) a countably infinite set $\mathcal{V}$ of variables ranged over by $a, b, c, \ldots$;

(2) a countably infinite set $\mathcal{M V}$ of meta-variables with fixed arities, written as $Z_{i}^{n}$ where $n$ is the arity of $Z_{i}^{n}$ (when there is no ambiguity, $n$ is omitted);

(3) an abstraction operator [.]·;

(4) function symbols $f, g, \ldots$ with fixed arities; and

(5) improper symbols '(', ')'and ','.

Definition 2.17 (Syntax). CRS meta-terms are generated by the grammar

$$
s, t::=a\left|Z_{i}^{n} t\right|[a] t|f t|\left(t_{1}, \ldots, t_{n}\right) \quad(n \geq 0)
$$

Only variables can be abstracted; in a function application $f t$ (resp. meta-application $\left.Z_{i}^{n} t\right)$, $t$ is a $n$-tuple respecting the arity of the function symbol $f$ (resp. meta-variable $Z_{i}^{n}$ ); when the arity is 0 , we omit the brackets in applications and meta-applications (so $Z_{i}^{0}$ is a metaterm). 
Definition 2.18. We write $M V(t)$ and $\operatorname{Var}(t)$ for the set of meta-variables and variables occurring in a meta-term $t$, respectively (the same notation is used for rules, etc.). They are inductively defined as follows:

$$
\begin{array}{llc}
M V(a)=\varnothing & M V\left(Z_{i}^{n} t\right)=M V(t) \cup\left\{Z_{i}^{n}\right\} & M V(f t)=M V(t) \\
M V([a] t)=M V(t) & M V\left(\left(t_{1}, \ldots, t_{n}\right)\right)=M V\left(t_{1}\right) \cup \cdots \cup M V\left(t_{n}\right) \\
\operatorname{Var}(a)=a & \operatorname{Var}\left(Z_{i}^{n} t\right)=\operatorname{Var}(t) \quad \operatorname{Var}(f t)=\operatorname{Var}(t) \\
\operatorname{Var}([a] t)=\operatorname{Var}(t) \cup\{a\} & \operatorname{Var}\left(\left(t_{1}, \ldots, t_{n}\right)\right)=\operatorname{Var}\left(t_{1}\right) \cup \cdots \cup \operatorname{Var}\left(t_{n}\right)
\end{array}
$$

In CRSs a distinction is made between meta-terms and terms. Meta-terms are the expressions built from the symbols in the alphabet, in the usual way (see Definition 2.17). Variables that occur in the scope of the abstraction operator are bound, and free otherwise. Meta-terms are defined modulo renaming of bound variables, that is, a meta-term represents an $\alpha$-equivalence class. Terms are meta-terms that do not contain meta-variables, and are also defined modulo $\alpha$-equivalence. A (meta-)term is closed if every variable occurrence is bound.

Definition 2.19. Let $s$ be a CRS meta-term. The set $\mathcal{P} o s(s)$ of positions in $s$ is a set of strings of positive integers, which is inductively defined as follows:

- if $s=a$, then $\operatorname{Pos}(s)=\{\epsilon\}$, where $\epsilon$ denotes the empty string;

- if $s=Z_{i}^{n} t$ then $\operatorname{Pos}(s)=\{\epsilon\} \cup\{1 \cdot p \mid p \in \operatorname{Pos}(t)\}$;

- if $s=[a] t$, then $\operatorname{Pos}(s)=\{\epsilon\} \cup\{1 \cdot p \mid p \in \operatorname{Pos}(t)\}$;

- if $s=f t$, then $\operatorname{Pos}(s)=\{\epsilon\} \cup\{1 \cdot p \mid p \in \mathcal{P}$ os $(t)\}$;

- if $s=\left(t_{1}, \ldots, t_{n}\right)$, then $\mathcal{P} o s(s)=\{\epsilon\} \cup \bigcup_{i=1}^{n}\left\{i \cdot p \mid p \in \mathcal{P}\right.$ os $\left.\left(t_{i}\right)\right\}$.

The position $\epsilon$ is called the root position of the term $s$, and the symbol at this position is called the root symbol of $s$.

Definition 2.20 (CRS rewrite rules). A rewrite rule is a pair of meta-terms, written $l \Rightarrow r$, where $l, r$ are closed, $l$ has the form $f\left(s_{1}, \ldots, s_{n}\right.$ ) where $n \geq 0$ (when $n=0$ we omit the parentheses), $M V(r) \subseteq M V(l)$, and $M V(l)$ occur only in the form $Z_{i}^{n}\left(a_{1}, \ldots, a_{n}\right)$, where $a_{1}, \ldots, a_{n}$ are pairwise distinct bound variables.

Example 2.21. The $\beta$-reduction rule for the $\lambda$-calculus is written:

$$
\operatorname{app}\left(\operatorname{lam}([a] Z(a)), Z^{\prime}\right) \Rightarrow Z\left(Z^{\prime}\right)
$$

where $Z$ is a unary meta-variable and $Z^{\prime}$ is 0 -ary.

The reduction relation is defined on terms. To extract from rules the actual rewrite relation, each meta-variable is replaced by a special kind of $\lambda$-term, and in the obtained term all $\beta$-redexes and the residuals of these $\beta$-redexes are reduced (i.e., a complete development is performed). Formally, the rewrite relation is defined using substitutes and valuations.

Definition 2.22 (Substitute). An $n$-ary substitute is an expression of the form $\underline{\lambda}\left(a_{1} \ldots a_{n}\right) . s$, where $s$ is a term and $a_{1}, \ldots, a_{n}$ are different variables bound in $\underline{\lambda}\left(a_{1} \ldots a_{n}\right) . s$. We use a meta-lambda $\underline{\lambda}$ to emphasise that this is part of the meta-language.

An $n$-ary substitute $\underline{\lambda}\left(a_{1} \ldots a_{n}\right) . s$ may be applied to a $n$-tuple $\left(t_{1}, \ldots, t_{n}\right)$ of terms, resulting in the following simultaneous substitution:

$$
\left(\underline{\lambda}\left(a_{1} \ldots a_{n}\right) . s\right)\left(t_{1}, \ldots, t_{n}\right)=s\left[a_{1} \mapsto t_{1}, \ldots, a_{n} \mapsto t_{n}\right]
$$


where we denote by $s[a \mapsto t]$ the capture-avoiding substitution of variable $a$ by term $t$ in the CRS term $s$.

Definition 2.23 (Valuation). A valuation $\sigma$ assigns an $n$-ary substitute to each $n$-ary meta-variable:

$$
\sigma\left(Z_{i}^{n}\right)=\underline{\lambda}\left(a_{1}, \ldots, a_{n}\right) . s .
$$

It is extended to a mapping from meta-terms to terms as follows:

(1) First, replace all meta-variables in the term for their images in $\sigma$ as shown below.

$$
\begin{aligned}
& \sigma(a)=a \text { for } a \in \mathcal{V} \quad \sigma([a] t)=[a] \sigma(t) \quad \sigma(f t)=f \sigma(t) \\
& \sigma\left(\left(t_{1}, \ldots, t_{n}\right)\right)=\left(\sigma\left(t_{1}\right), \ldots, \sigma\left(t_{n}\right)\right) \quad \sigma\left(Z_{i}^{n} t\right)=\sigma\left(Z_{i}^{n}\right) \sigma(t) .
\end{aligned}
$$

(2) Next, perform the developments of the $\beta$-redexes created.

Valuations must satisfy some safety conditions. Before stating the conditions, we recall a standard naming convention used in CRSs, originally stated by Barendregt for the $\lambda$ calculus [2].

Remark 2.24 (Barendregt's variable convention). CRSs adopt the following naming conventions:

- all bound variables are chosen to be different among them, that is, each binder uses a distinct variable name;

- bound variables are also chosen to be different from free variables.

In CRSs, rewriting is performed under the following conditions.

Definition 2.25 (Safety conditions). The CRS rule $l \Rightarrow r$ is safe for the valuation $\sigma$ if free variables occurring in substitute $\sigma(Z)$ are different from the bound variables in both $l, r$ for all $Z \in \operatorname{dom}(\sigma)$ and also, we say $\sigma$ is safe with respect to itself when there are no two substitutes $\sigma(Z), \sigma\left(Z^{\prime}\right)$ where a free variable in $\sigma(Z)$ occurs bound in $\sigma\left(Z^{\prime}\right)$ or vice versa.

In the rest of the paper we adopt, without loss of generality, Barendregt's convention for CRSs and assume that all valuations are safe with respect to themselves and the reduction rules to which they are applied.

A context is a term with an occurrence of a special symbol [] called hole. A rewrite step is now defined in the usual way.

Definition 2.26 (Rewrite step). Let $\sigma$ be a valuation and $C$ [] a context. If $l \Rightarrow r$ is a rewrite rule, then $C[\sigma(l)] \Rightarrow C[\sigma(r)]$ is a rewrite (or reduction) step.

Example 2.27. The following is a rewrite step using the $\beta$-rule given in Example 2.21 ,

$$
\operatorname{app}(\operatorname{lam}([a] f(a, a)), t) \Rightarrow_{\beta} f(t, t) \text {. }
$$

To generate the reduction, a valuation $\sigma$ that maps $Z$ to $\underline{\lambda}(b) \cdot f(b, b)$ and $Z^{\prime}$ to the term $t$ is applied to the rule. Then, $\sigma\left(\operatorname{app}\left(\operatorname{lam}([a] Z(a)), Z^{\prime}\right)\right)$ is the term $\operatorname{app}(\operatorname{lam}([a] f(a, a)), t)$ obtained by first replacing $Z$ and $Z^{\prime}$ as indicated by $\sigma$ and then reducing the $\beta$-redex $(\underline{\lambda}(b) . f(b, b))(a)$. Also, $\sigma\left(Z\left(Z^{\prime}\right)\right)$ is the term $f(t, t)$ obtained by first replacing $Z$ and $Z^{\prime}$, resulting in $(\underline{\lambda}(b) . f(b, b))(t)$, and then $\beta$-reducing to $f(b, b)\{b \mapsto t\}$. 
2.3. Symmetric groups. The following definitions and properties, well-known in group theory [33], will be useful later, in Section 6, when translating CRSs into NRSs.

Definition 2.28. The symmetric group $\mathcal{S}_{n}$ is the group of bijections (permutations) of $\left\{a_{1}, \ldots, a_{n}\right\}$ to itself. A standard notation for the permutation that maps $a_{i}$ to $\pi\left(a_{i}\right)$ is the two-line notation or array form

$$
\left(\begin{array}{ccccc}
a_{1} & a_{2} & a_{3} & \ldots & a_{n} \\
\pi\left(a_{1}\right) & \pi\left(a_{2}\right) & \pi\left(a_{3}\right) & \ldots & \pi\left(a_{n}\right)
\end{array}\right)
$$

Under composition of mappings, the permutations of $\left\{a_{1}, \ldots, a_{n}\right\}$ are a group.

A permutation $\pi \in \mathcal{S}_{n}$ is a $k$-cycle if there are distinct elements $a_{1}, a_{2}, \ldots, a_{k}$ such that $\pi\left(a_{1}\right)=a_{2}, \pi\left(a_{2}\right)=a_{3}, \ldots, \pi\left(a_{k}\right)=a_{1}$ and $\pi$ fixes every other element. A 2-cycle permutation is known as a transposition, or swapping. There is a standard notation for $k$-cycle forms:

$$
\left(a_{1}, a_{2}, a_{3}, \ldots, a_{k}\right)
$$

A pair of cycles $\left(a_{1}, \ldots, a_{n}\right)$ and $\left(a_{1}^{\prime}, \ldots, a_{n}^{\prime}\right)$ are disjoint when the sets $\left\{a_{1}, \ldots, a_{n}\right\}$ and $\left\{a_{1}^{\prime}, \ldots, a_{n}^{\prime}\right\}$ are disjoint.

Lemma 2.29. Every permutation is uniquely expressible as a product of disjoint cycles.

Disjoint cycles commute.

Theorem 2.30 (Product of transpositions). Every permutation in $\mathcal{S}_{n}, n>1$, can be expressed as a product of 2-cycles.

Theorem 2.30justifies our choice of representation for permutations as lists of swappings in Section 2.1.

Property 2.31 ( $k$-cycle as a product of transpositions). A $k$-cycle $\left(a_{1}, a_{2}, \ldots, a_{k-1}, a_{k}\right)$ in $\mathcal{S}_{n}$ can be decomposed into transpositions:

$$
\left(a_{1}, a_{2}, \ldots, a_{k-1}, a_{k}\right)=\left(a_{1} a_{k}\right)\left(a_{1} a_{k-1}\right) \ldots\left(\begin{array}{ll}
a_{1} & a_{2}
\end{array}\right)
$$

following the grammar of permutations given in Section 2.1.

Using this property, when given a permutation defined as a bijection, we can find its corresponding list of swappings by first writing it as a product of disjoint cycles, and then decomposing each cycle into 2-cycles as shown in the example below.

Example 2.32. The following bijective function:

$$
\begin{array}{lll}
f(a)=c & f(b)=d & f(c)=e \\
f(d)=f & f(e)=g & f(f)=h \\
f(g)=a & f(h)=b & f(i)=i
\end{array}
$$

has the following array form associated with it.

$$
f=\left(\begin{array}{lllllllll}
a & b & c & d & e & f & g & h & i \\
c & d & e & f & g & h & a & b & i
\end{array}\right)
$$

In this case, the first row represents the elements in the domain, in lexicographic order, and the second row their respective image.

To convert array form notation into cycle notation we follow these steps: 
- Start with the smallest letter in the set, in this case $a$, since $f(a)=c$ we begin the cycle by writing

$$
(a, c, \ldots) \ldots
$$

Notice we could start with any letter since there are a number of equivalent representations of $f$ in cycle form. Non-unique representation does not alter the action of the permutations in $f$.

- Next, $c$ maps to $e$, so we continue building the cycle

$$
(a, c, e, \ldots) \ldots
$$

- Continuing in this way we construct $(a, c, e, g, \ldots) \ldots$ and since $g$ maps back to $a$, then we close off the cycle

$$
(a, c, e, g) \ldots
$$

- Next, we pick the smallest letter that does not appear in any previously constructed cycle, this is letter $b$ in this case, and repeat the previous steps to construct a new cycle:

$$
(a, c, e, g)(b, d, f, h) \ldots
$$

- Finally the last letter $i$ is picked and the cycle is constructed. In this case $i$ maps to itself:

$$
(a, c, e, g)(b, d, f, h)(i)
$$

- Decomposition into 2-cycle form by application of Property 2.31 produces:

$$
(b h)(b f)(b d)(a g)(a e)(a c)
$$

where the 1-cycle $(i)$ is discarded since it produces $(i i)$.

This simple method of converting bijective mappings in array form into a 2-cycle representation of permutations, while preserving the action of the mappings, is instrumental for a correct translation of meta-applications in CRSs into permutations suspended on variables in NRSs. We postpone further discussion along with the formal definition of the conversion procedure for Section 6, where CRS rules and terms are translated to NRSs.

\section{Translating from Nominal to CRS Syntax}

In this section, we give an overview of the main issues surrounding the translation between NRS and CRS syntax, along with our approach to solve them. Further examples and formal proofs are given after defining the translation function.

3.1. Overview of the Problem. In order to design a function that transforms NRSs to CRSs, we must take into account the following distinctions between formalisms:

- CRS rules are closed by definition, but this is not the case for nominal rules. Thus, the translation is restricted to closed NRS rules.

- CRSs make a distinction between meta-terms and terms and rewriting is defined only on terms. Such a distinction does not occur in NRSs. To solve such issue, nominal rewriting is restricted to operate only on ground terms.

- NRSs contain a (possibly empty) set of freshness assumptions to avoid accidental name capture. Such a mechanism does not exist in CRSs, where (meta-)terms are defined modulo $\alpha$. Therefore, freshness assumptions must be considered when constructing both meta-applications and CRS substitutes. 
- Nominal variables have arity zero whereas CRS meta-variables may have non-zero arity. Hence, a unique arity for all occurrences of a meta-variable must be correctly enforced when applying the translation function.

- A moderated variable $\pi \cdot X$ contains a suspended permutation $\pi$ which is applied immediately after instantiation of $X$. There are no permutations in CRSs. Intuitively, we must observe the potential effects of applying the permutation to any instantiation of $X$ and translate accordingly to CRSs to simulate the action of $\pi$.

- Nominal substitution allows capture, whereas substitution in CRSs is non-capturing. Therefore, the translation algorithm must recognise abstractions and build CRS substitutes that replicate the behaviour of the substitutions in NRSs.

First, we discuss the simulation of capturing substitution in CRSs. For this, we use an auxiliary function $\Lambda$ which traverses a nominal term $t$ and outputs, for each nominal variable $X$ in $t$, a set of distinct atoms that occur abstracted above any of the occurrences of $X$ in $t$. For instance, if $t=g([a][b] X,[a][b] X)$ then $\Lambda_{t}(X)=\{a, b\}$. Atoms in $\Lambda_{t}(X)$ would be captured if $X$ is instantiated by a term that contains these atoms free, e.g.: $\sigma(X)=f(a, b)$. However, in CRSs, a distinct representative of the term class would be chosen if any of the $a, b$ variables were to appear free in a substitute. Since variable capture must be allowed, $\Lambda_{t}(X)$ is used to create the variable binding list for a substitute of a meta-variable $X$, in the case of the example, $\sigma=[X \mapsto \underline{\lambda}(a . b) . f(a, b)]$. Moreover, as CRS rules must be closed, $\Lambda_{t}(X)$ also aids in constructing the list of bound variables (thus occurring in $\Lambda_{t}(X)$ ) associated with each occurrence of a meta-variable $X$ in a rule. Therefore, the example above would translate to $\hat{t}=g([a][b] X(a, b),[a][b] X(a, b))$. Indeed, our translation algorithm outputs closed CRS meta-terms when applied to closed nominal terms.

Additionally, when translating a term-in-context $\Delta \vdash t$, we take into account the freshness constraints in $\Delta$ such that, if $a \in \Lambda_{t}(X)$ and $a \# X \in \Delta$, then $a$ is not considered when simulating variable capture in CRSs, since $a$ cannot occur free in any substitution $\sigma$ for $X$. However, there are some special cases. The application of a permutation $\pi$ to $\sigma$ after instantiation may alter the final outcome of the instantiation. CRSs rely on the list of variable arguments and binders along with the mechanism of $\beta$-reduction to simulate the process of swapping atoms. This means that variables that were not initially part of the argument list are now introduced back into the meta-application in order to deal with the necessary renamings.

In our translation, the list of variable arguments occurring in each meta-application is ordered with respect to a total ordering 1 By doing so, there is no relation between the position of each variable in the argument list of the meta-application and the position of the abstractions in the CRS meta-term. On the other hand, there is a bijection between the variables in the binding list added to the substitute for $X$ and the variables in the argument list, as expected. Furthermore, when translating nominal substitutions, we will show that, for all occurrences of a meta-application, each substitute built by the translation function is equivalent modulo $\alpha$. This property allows us to choose just one of the occurrences (we choose the leftmost one) to work with and then apply it back to all the occurrences in the translated CRS term. We give examples below.

Permutations are the main cause of variations among occurrences of the same variable in a term when instantiated, leading to possible modifications of the binding structure.

\footnotetext{
${ }^{1}$ We have chosen a lexicographic ordering but any other total ordering also works.
} 
Consider for example,

$$
t=\vdash f([a][b] X,[a][b](a b) \cdot X)
$$

and the substitution $\sigma=[X \mapsto g(a, b)]$, which produces the term

$$
t \sigma=f([a][b] g(a, b),[a][b] g(b, a))
$$

where atoms $a, b$ have been swapped on the second occurrence of $X$. How should we take into account these permutations in the CRS syntax?.

Intuitively, we apply each $\pi$ directly to the set of atoms $\Lambda_{t}(X)=\{a, b\}$, for each occurrence of $\pi \cdot X$ in $t$, resulting in two argument lists: $(a, b)$ for the first occurrence of $X$ and $(b, a)$ for the second one. However, this approach is not effective when we encounter occurrences of $\pi \cdot X$ where swappings in $\pi$ contain atoms which do not occur abstracted above $X$, therefore not contained in $\Lambda$. Take for instance the nominal term

$$
s=[a](a b) \cdot X
$$

where $\Lambda_{s}(X)=\{a\}$. A direct application of $\pi$ to $\Lambda_{s}(X)$ results in the CRS meta-term

$$
\hat{s}=[a] X(b) .
$$

We immediately notice two problems with this translation: the possibility of atom $b$ occurring in an instantiation of $X$ in $s$ has not been accounted for in the CRS translation $\hat{s}$. We expect $b$ to be renamed to $a$ by the swapping $(a b)$ and captured by the abstraction in $s$, yet $a$ does not appear in the variable argument list after application of $\pi$ to $\Lambda_{s}(X)$ in $\hat{s}$. Furthermore, if our goal is to translate NRS rules into CRS rules, and CRS rules are closed by definition, the application of $\pi$ to $\Lambda_{s}(X)$ produces a list of atoms no longer bound above $X$, and thus not suitable for CRSs.

Alternatively, we order lexicographically the set $\pi^{-1} \cdot \Lambda_{s}(X)$, obtaining an intermediate list: $x s$. It contains those atoms that could be captured if occurring free in a substitution for $X$ (notice in the example $x s=\{b\}$ where $b$ is captured because of $(a b)$ ). In other words, $x s$ is the initial list of binders that allow our translation to capture variables. Next, $\pi$ is applied to $x s$ to return an ordered and filtered version of $\Lambda_{s}(X)$ as a list, which we call $\overline{x s}$ (in the example, $\overline{x s}=a$ ). The list $\overline{x s}$ contains variables occurring in $\Lambda$ and thus bound so that it can finally be displayed as the variable argument list for the meta-application of an occurrence of $X$. Therefore, at this point we have a bijection from the atoms in $x s$ to those in $\overline{x s}$ such that $x s_{i} \mapsto \overline{x s}_{i}$ is the mechanism that maps a captured variable $a \in x s$ at position $i$ to the variable $\pi(a) \in \overline{x s}$ at position $i$ when a substitution is provided. In addition, $\pi$ must also be applied to the nominal substitution $\sigma(X)$ prior translation, to rename atoms in support $(\pi)$ not in scope of $\Lambda_{t}(X)$. As a result, $\pi$ is also applied to the binding list $x s, \pi \cdot x s$, to preserve the original binding structure when added to $\pi \cdot \sigma(X)$, i.e., $\underline{\lambda}(\pi \cdot x s) \cdot(\pi \cdot \sigma(X))$. Note that $\pi \cdot x s$ is $\overline{x s}$.

A more detailed explanation of the algorithm is provided after its definition. Now, we look at the examples again, applying the new approach:

$$
(t=\vdash f([a][b] X,[a][b](a b) \cdot X), \sigma=[X \mapsto g(a, b)])
$$

is translated as

$$
(\hat{t}=f([a][b] X(a, b),[a][b] X(b, a)), \hat{\sigma}=[X \mapsto \underline{\lambda}(a \cdot b) \cdot g(a, b)])
$$

since the leftmost one is chosen, where

$$
\hat{\sigma}(\hat{t})=f([a][b] g(a, b), \quad[a][b] g(b, a))
$$


Also,

translates to the CRS meta-term

$$
s=[a](a b) \cdot X
$$

$$
\hat{s}=[a] X(a)
$$

which is now closed. And a nominal substitution $\sigma=[X \mapsto g(a, b))]$ to instantiate nominal term $s$ would translate into the valuation $\hat{\sigma}=[X \mapsto \underline{\lambda}(a) \cdot g(b, a)]$.

We have discussed the main issues in the translation of NRSs to CRSs, together with strategies to solve such issues. In the rest of the section we formalise this approach and provide examples.

3.2. Translating Nominal Terms. For each nominal signature $\Sigma$, and sets $\mathcal{A}$ and $\mathcal{X}$ of atoms and variables, we consider a CRS alphabet containing $\Sigma$, variables $\mathcal{A}$ and metavariables $\mathcal{X}$.

First we define an auxiliary function, $\Lambda$, to compute, for each nominal term $t$, and each variable $X$ in $t$, the set of atoms that may be captured when $X$ is instantiated.

Intuitively, $\Lambda_{t}(X)=\left\{a_{1}, \ldots, a_{n}\right\}$ if $X \in V(t)$ has $k$ occurrences in $t, A_{i}$ is the set of atoms abstracted above the $i$ th occurrence of $X$, and $\left\{a_{1}, \ldots, a_{n}\right\}=A_{1} \cup \ldots \cup A_{k}$. In other words, $\Lambda_{t}(X)$ is the set of all the atoms abstracted above occurrences of $X$ in $t$.

Definition 3.1 (Mapping $\Lambda_{t}$ ). For each nominal term $t$, the mapping $\Lambda_{t}: V(t) \rightarrow \mathcal{P}(\mathcal{A})$ is defined by $\Lambda_{t}(X)=\Lambda^{\prime}(\varnothing, t)(X)$, where $\Lambda^{\prime}(\cdot, \cdot)$ is an auxiliary function defined inductively over the structure of $t$ as follows:

$$
\begin{array}{ll}
\Lambda^{\prime}(A, a)(X) & =\varnothing, \\
\Lambda^{\prime}(A, \pi \cdot X)(X) & =A, \\
\Lambda^{\prime}(A, \pi \cdot Y)(X) & =\varnothing, \\
\Lambda^{\prime}(A,[a] s)(X) & =\Lambda^{\prime}(A \cup\{a\}, s)(X), \\
\Lambda^{\prime}(A, f s)(X) & =\Lambda^{\prime}(A, s)(X), \\
\Lambda^{\prime}\left(A,\left(s_{1}, \ldots, s_{n}\right)\right)(X) & =\Lambda^{\prime}\left(A, s_{1}\right)(X) \cup \ldots \cup \Lambda^{\prime}\left(A, s_{n}\right)(X) .
\end{array}
$$

For example, if $t=([a] X,[b] X,[c] Y)$ then $\Lambda_{t}(X)=\{a, b\}$ and $\Lambda_{t}(Y)=\{c\}$.

Next, we define the translation of nominal terms into CRS (meta-)terms.

Definition 3.2 (Term translation). Let $\Delta \vdash t$ be a nominal term-in-context and $\Lambda_{t}$ as in Definition 3.1. Then $\mathcal{T}(\Delta, t)=\llbracket t \rrbracket_{\Lambda_{t}}^{\Delta}$, where $\llbracket \cdot \rrbracket_{\Lambda_{t}}^{\Delta}$ is an auxiliary function defined by induction over the structure of nominal terms as follows:

$$
\begin{aligned}
& \text { (atom) } \llbracket a \rrbracket_{\Lambda_{t}}^{\Delta} \quad=a, \\
& \text { (var) } \llbracket \pi \cdot X \rrbracket_{\Lambda_{t}}^{\Delta} \quad=X(\overline{x s}) \text { where }
\end{aligned}
$$

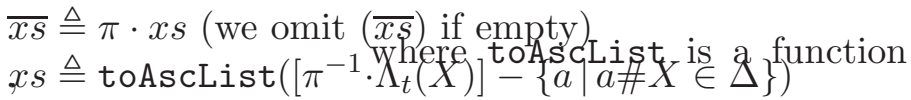

$$
\begin{aligned}
& \text { (abs) } \quad \llbracket[a] s \rrbracket_{\Lambda_{t}}^{\Delta} \quad=[a] \llbracket s \rrbracket_{\Lambda_{t}}^{\Delta}, \\
& \text { (fun) } \llbracket f s \rrbracket \Delta_{\Lambda_{t}}^{\Lambda_{t}} \quad=f \llbracket s \rrbracket \Delta_{\Lambda_{t}}, \\
& \text { (tuple) } \llbracket\left(s_{1}, \ldots, s_{n}\right) \rrbracket_{\Lambda_{t}}^{\Delta}=\left(\llbracket s_{1} \rrbracket_{\Lambda_{t}}^{\Delta}, \ldots, \llbracket s_{n} \rrbracket_{\Lambda_{t}}^{\Delta_{t}}\right) .
\end{aligned}
$$

that builds a sorted list2 from a set of atoms. When there is no ambiguity, we refer to the translation of a term $t$ as $\hat{t}$.

\footnotetext{
${ }^{2}$ List of atoms in ascending lexical order.
} 
The interesting case in the translation is that of a variable. Intuitively, the list $x s$ contains atoms $a_{i}$ that will be captured if occurring free in any instance of $X$, because $\pi \cdot X$ occurs in the scope of an abstraction for $\pi \cdot a_{i}$. Note that if $a \notin \overline{x s}$ then if $\pi^{-1} \cdot a$ is in a substitution for $X$, then $a$ will be in $\operatorname{supp}(t)$ (it will not be captured). In other words, $a \notin \overline{x s}$ implies that if $\pi^{-1} \cdot a \in \operatorname{supp}(X)$ then $a \in \operatorname{supp}(t)$.

The examples below illustrate the translation of nominal terms to CRS terms. Prior to that, we highlight a property of the translation and introduce some terminology.

Lemma 3.3 (Preservation of variables as meta-variables in the translation). Suppose $\Delta \vdash t$ is a term-in-context and $\hat{t}=\llbracket t \rrbracket_{\Lambda_{t}}^{\Delta}$ its translation by Definition 3.2. Then, $\pi \cdot X$ occurs in $t$ at position $p$ if and only if $X(\overline{x s})$ occurs in $\hat{t}$ at position $p$ for some $\overline{x s}$.

Proof. By induction on the structure of $t$ and the fact that there is a one-to-one correspondence between the elements of $t$ and $\hat{t}$ as shown in the syntax-directed translation function in Definition 3.2, namely a variable $\pi \cdot X$ occurs at position $p$ in $t$ if and only if there exists a meta-variable $X$ at $p$ in its translation $\hat{t}$ along with a list of variable arguments $\overline{x s}$.

Remark 3.4 (Translation of a moderated variable). Let $\mathcal{T}(\Delta, t)=\hat{t}$, where $\Delta \vdash t$ is a term-in-context and $\mathcal{T}$ is the translation function given in Definition 3.2. If $\pi \cdot X$ occurs in $t$ at position $p$ then $X(\overline{x s})=\llbracket \pi \cdot X \rrbracket_{\Lambda_{t}}^{\Delta}$ occurs in $\hat{t}$ at position $p$, by Lemma 3.3. We say that $X(\overline{x s})$ is the translation of $\pi \cdot X$ in $\hat{t}$ and call $\overline{x s}$ the arguments of $X$.

Example 3.5. According to Definition 3.2 , the (closed) nominal term $\vdash[a][b] X$ is translated as the CRS meta-term $[a][b] X(a, b)$, where we include both variables in the meta-application as they may appear free in a substitution for $X$.

Freshness constraints are taken into account in the translation of variables. Consider $a \# X \vdash[a][b] X$, which is translated to the CRS meta-term $[a][b] X(b)$. Since any substitution for $X$ must satisfy $\Delta$ (that is, $\vdash a \# X \sigma$ ), $a$ is not included in the arguments of $X$.

However, a freshness constraint does not always produce this effect, it depends on the permutations in the term. We adjust our example to show this. Consider

$$
a \# X \vdash[a][b](a b) \cdot X .
$$

In this case we should take into account the mapping $b \mapsto a$ but not $a \mapsto b$ since $a \# X \in \Delta$. Our translation outputs the CRS meta-term

$$
[a][b] X(a),
$$

which suggests that $a$ may occur free in an instance of $X$ contradicting the nominal constraint $a \# X \in \Delta$. However, since any nominal substitution $\sigma$ that instantiates $X$ must also satisfy $\Delta$, the atom $a$ does not occur unabstracted in $X \sigma$ or in its CRS translation. Hence the mapping $a \mapsto b$ is discarded.

Example 3.6. Let $t$ be the (closed) nominal term $\vdash[a][b](a c) \cdot X$, where $\Delta=\varnothing$. Then $\Lambda_{t}(X)=\{a, b\}, x s=[b, c]$, and $\overline{x s}=[b, a]$. The swapping $(a c)$ maps the abstracted atom $a$ to the unabstracted atom $c$. This particular kind of mapping cannot be explicitly represented at term level thus it will be applied to the substitute that instantiates $X$, if any. This is shown in more detail in Section 4 when describing the translation of substitutions. The translation function for terms (Definition 3.2) produces the meta-term

$$
[a][b] X(b, a)
$$

which effectively takes into account the rest of the mappings in the permutation, that is, $b \mapsto b, c \mapsto a$, generating a closed CRS meta-term. 
The following example illustrates the case of a term with multiple occurrences of a variable with different suspended permutations.

Example 3.7. The translation of the (closed) nominal term-in-context

$$
f \# X \vdash g([a][b][c][d](c f)(a b) \cdot X,[a][b][c][d](c f)(d b)(a d) \cdot X)
$$

is the CRS meta-term

$$
g([a][b][c][d] X(b, a, d),[a][b][c][d] X(b, d, a))
$$

where

- $\Lambda_{t}(X)=\{a, b, c, d\}$,

- $x s_{1}=x s_{2}=[a, b, d]$ (the sub-indices are used to refer to the two occurrences of $X$ ),

- $\pi_{1} \cdot x s_{1}=\overline{x s}_{1}=[b, a, d]$ and $\pi_{2} \cdot x s_{2}=\overline{x s}_{2}=[b, d, a]$

Notice that if atom $c$ occurs unabstracted in an instance $X \sigma$ of $X$, there exists a mapping $c \mapsto f$ in both permutations that renames $c$ to $f$ when translating $X \sigma$. This renaming cannot be dealt with at term level because it would involve including variable $f$ in the list of arguments and the result would not be closed. However, the function that translates nominal substitutions (see Definition 5.1) takes care of this renaming.

The following lemma states the uniqueness of the intermediate list of atoms $x s$ computed by the translation function for each of the occurrences of a variable $X$ in a closed nominal term. This is important when proving preservation of arities among meta-variable occurrences and closedness of the translated CRS meta-term.

Lemma 3.8 (Equivalence). Let $\Delta \vdash t$ be a closed term-in-context and $\mathcal{T}(\Delta, t)=\hat{t}$ its CRS translation. If $\pi_{1} \cdot X$ and $\pi_{2} \cdot X$ are two occurrences of the same variable $X$ in $t$, and $X\left(\overline{x s_{1}}\right), X\left(\overline{x s_{2}}\right)$ are their respective translations in $\hat{t}$, then $\pi_{1}^{-1} \cdot \overline{x s_{1}}=\pi_{2}^{-1} \cdot \overline{x s_{2}}$.

Proof. This is a consequence of the definition of $\overline{x s}$ and the fact that the term is closed. More precisely, by Definition 3.2, the translation of $\pi \cdot X$ is $X(\overline{x s})$ where $\overline{x s} \triangleq \pi \cdot x s$ and $x s \triangleq \operatorname{toAscList}\left(\left[\pi^{-1} \cdot \Lambda_{t}(X)\right]-\{a \mid a \# X \in \Delta\}\right)$.

It is sufficient to prove that if an atom $a \in x s_{1}$ at a position $i$ then $a \in x s_{2}$ at a position $j$ such that $i=j$, and vice versa.

Now, for any $a$ such that $a \in x s_{1}$, it is also the case that $\pi_{1}(a) \in \Lambda_{t}(X)$ (since $\pi^{-1} \circ \pi \cdot a=$ a) and $a \# X \notin \Delta$ by definition of $x s$, then either

(1) $\pi_{2}(a) \notin \Lambda_{t}(X)$, or

(2) $\pi_{2}(a) \in \Lambda_{t}(X)$, thus $a \in x s_{2}$,

No other cases are possible.

In case (1), any substitution of $X$ containing atom $a$ unabstracted is in the scope of an abstraction in $\pi_{1} \cdot X$ since $\pi_{1}(a) \in \Lambda_{t}(X)$, but unabstracted under $\pi_{2} \cdot X$, since $\pi_{2}(a) \notin \Lambda_{t}(X)$. Since the term is closed it must be the case that $a \# X \in \Delta$ by Definition 2.14, contradicting the fact that $a \in x s_{1}$. Hence it is the case that $\pi_{2}(a) \in \Lambda_{t}(X)$ too, as stated in (2). Thus, we have established that for each $a$ in $x s_{1}$ at some position $i, a \in x s_{2}$ at some position $j$. Similarly, we can prove $a \in x s_{2}$ implies $a \in x s_{1}$. Since toAscList is applied to both $x s_{1}, x s_{2}$, then $i=j$ leading to $x s_{1}=x s_{2}$.

Therefore, for any pair $X\left(\overline{x s}_{1}\right), X\left(\overline{x s}_{2}\right)$ in $\hat{t}, \pi_{1}^{-1} \cdot \overline{x s}_{1}=\pi_{2}^{-1} \cdot \overline{x s}_{2}$. 
Next we prove that the translation function produces CRS (meta-)terms (in particular, meta-applications respect variable arities).

Property 3.9 (Arity). Let $\Delta \vdash t$ be a closed term-in-context and $\mathcal{T}(\Delta, t)=\hat{t}$ its $C R S$ translation. For each occurrence of $X$ in $t$, there is a corresponding occurrence of $X$ in $\hat{t}$; moreover, there exists $n$ (the arity of $X$ ) such that all the occurrences of $X$ in $\hat{t}$ are in meta-applications of arity $n$. In other words, in the translated term all the occurrences of $X$ respect the arity of $X$.

Proof. The translation is syntax directed. For every $\pi \cdot X$ in $t, \llbracket \pi \cdot X \rrbracket_{\Lambda_{t}}^{\Delta}=X(\overline{x s})$ where, by Property $3.8, \pi^{-1} \cdot \overline{x s}$ is a unique list of variables for all occurrences of $X(\overline{x s})$ in $\hat{t}$. This leads to $\overline{x s}$ having the same length $n$ for all $X$ in $\hat{t}$, where $n$ is the arity of $n$.

\section{Property 3.10 (Preservation of closedness).}

(a) If $\Delta \vdash t$ is a closed nominal term then its CRS translation $\hat{t}$ (according to Definition 3.2) is a closed CRS meta-term.

(b) Moreover, if the nominal term $t$ is ground, then its translation is a CRS term.

Proof. First we prove that the translation $\hat{t}$ is a CRS meta-term. This is due to:

(1) Our translation respecting the structure of $t$, which maps atoms to variables, moderated variables to meta-applications consisting of a meta-variable and its corresponding list of arguments in the form $X^{n}\left(a_{1}, \ldots, a_{n}\right)$ (where the arity $n \geq 0$ can be read directly from the meta-term and thus omitted), nominal abstraction to CRS abstraction, nominal functions to CRS functions and nominal tuples to CRS tuples.

(2) As a direct consequence of Properties 3.8 and 3.9, every meta-application $X^{n}(\overline{x s})$ respects the arity $n=|\overline{x s}|$ for all occurrences of $X$ in $\hat{t}$.

Hence, if $t$ is ground, $\hat{t}$ is a CRS term.

It remains to prove that $\hat{t}$ is closed. By definition of closedness of a nominal term, see Definition 2.14, every occurrence of an atom in $t$ is in the scope of an abstraction, since our translation respects the structure of $t$, see (1). Finally, we prove that variable arguments in a meta-application are bound. For this we show that for all occurrences of $X(\overline{x s})$ in $\hat{t}$, $\overline{x s}$ is a list of bound variables: by definition of $\mathcal{T}(\Delta, t)$ we know that $\overline{x s}=\pi \cdot x s$ for some $\pi$ such that $\pi \cdot X$ occurs in $t$. Also, $x s \triangleq \operatorname{toAscList}\left(\left[\pi^{-1} \cdot \Lambda_{t}(X)\right]-\{a \mid a \# X \in \Delta\}\right)$, that is, for each $a \in x s$ it is a requirement that $\pi(a) \in \Lambda_{t}(X)$ (since $\pi^{-1} \circ \pi \cdot a=a$ ). If $\pi(a) \in \Lambda_{t}(X)$, by Definition [3.1, $\pi(a)$ must occur abstracted above $X$. Moreover, as a consequence of Property 3.8, this is the case for all occurrences of $X$, otherwise $a \# X \in \Delta$ leading to $\pi(a) \notin \overline{x s}$.

Therefore all the variables in $\overline{x s}$ are bound, for all occurrences of $X(\overline{x s})$ in $\hat{t}$. Hence we conclude that $\hat{t}$ is a closed meta-term.

The following auxiliary lemmas are used to prove that $\alpha$-equivalent closed nominal terms have the same CRS translation. Intuitively this is the case since CRS terms are, by definition, considered modulo $\alpha$.

Lemma 3.11. If $\Delta \vdash s \approx_{\alpha} t$ and $\Delta \vdash s, \Delta \vdash t$ are closed, then for any variable $X$ such that $\pi_{s} \cdot X$ occurs in $s$ and $\pi_{t} \cdot X$ occurs in $t, \pi_{s}^{-1} \cdot \overline{x s}_{s}=\pi_{t}^{-1} \cdot \overline{x s}_{t}$, where $X\left(\overline{x s}_{s}\right)$ and $X\left(\overline{x s}_{t}\right)$ are the translations of $\pi_{s} \cdot X$ in $s$ with arguments $\overline{x s}_{s}$ and $\pi_{t} \cdot X$ in $t$ with arguments $\overline{x s}$, respectively.

Proof. Direct consequence of Lemma 3.8 and the fact that $\alpha$-equivalent terms have the same support. 
Lemma 3.12. Let $\Delta \vdash s$ be a nominal term-in-context and $\Lambda_{s}$ as defined in Definition 3.1. Then $\llbracket(a b) \cdot s^{\prime} \rrbracket_{(a b) \cdot \Lambda_{s}}^{\Delta}=\llbracket s^{\prime} \rrbracket_{\Lambda_{s}}^{\Delta}\{a \mapsto b, b \mapsto a\}$ for all subterms $s^{\prime}$ of $s$.

Proof. By induction on the structure of $s^{\prime}$.

- The case $\left(s^{\prime}=c\right)$. There are three cases to consider: $c=a, c=b$, and $c \neq a, c \neq b$.

- Suppose $c=a$. Then, by Definition [3.2. $\llbracket b \rrbracket_{(a b) \cdot \Lambda_{s}}^{\Delta}=b=\llbracket a \rrbracket_{\Lambda_{s}}^{\Delta}\{a \mapsto b, b \mapsto a\}$.

- Suppose $c=b$. Then, by Definition 3.2, $\llbracket a \rrbracket_{(a \quad b) \cdot \Lambda_{s}}^{\Delta}=a=\llbracket b \rrbracket_{\Lambda_{s}}^{\Delta}\{a \mapsto b, b \mapsto a\}$.

- Suppose $c \neq a$ and $c \neq b$. By Definition [3.2, $\llbracket c \rrbracket_{(a b) \cdot \Lambda_{s}}^{\Delta}=c=\llbracket c \rrbracket_{\Lambda_{s}}^{\Delta}\{a \mapsto b, b \mapsto a\}$.

- The case $\left(s^{\prime}=\pi \cdot X\right)$. By Definition [3.2, we have $\llbracket(a b) \cdot(\pi \cdot X) \rrbracket_{(a b) \cdot \Lambda_{s}}^{\Delta}=\llbracket\left(\left(\begin{array}{ll}a & b\end{array}\right) \circ\right.$ $\pi) \cdot X \rrbracket_{(a b) \cdot \Lambda_{s}}^{\Delta}=X(\overline{x s})$ and also $\llbracket \pi \cdot X \rrbracket_{\Lambda_{s}}^{\Delta}\{a \mapsto b, b \mapsto a\}=X\left(\overline{x s^{\prime}}\{a \mapsto b, b \mapsto a\}\right)$.

For this case we must take into account each atom $c \in \overline{x s}$ at a position $p$, then prove it also occurs at $p$ in the argument list $\overline{x s^{\prime}}\{a \mapsto b, b \mapsto a\}$.

We consider first the case where $\overline{x s}=\varnothing$ then the case where $\overline{x s} \neq \varnothing$.

(1) For the case where $\overline{x s}=\varnothing$, it is also the case that $(a b) \cdot \Lambda_{s}(X)=\varnothing$. Hence $\overline{x s^{\prime}}\{a \mapsto b, b \mapsto a\}=\varnothing$ by Definition 3.2 and the result follows.

(2) For the case where $\overline{x s} \neq \varnothing$, we distinguish cases depending on whether $c=a$, $c=b$ and finally for any other atom $c$ such that $a \neq c \neq b$. Notice that for any atom $c$, if $c \in \overline{x s}$ at $p$ then, observing Definition $3.2, \overline{x s}=((a b) \circ \pi) \cdot x s$ such that, by property of permutations, $\left(\pi^{-1} \circ(a b)\right) \cdot \overline{x s}=x s$ thus $\left(\pi^{-1} \circ(a b)\right) \cdot c \in x s$ also at $p$. Hence, $c \in(a b) \cdot \Lambda_{s}(X)$ and $\left(\pi^{-1} \circ(a b)\right) \cdot c \# X \notin \Delta$. This remark is implicitly applied in the three cases below.

- Suppose $c=a$ such that $a \in \overline{x s}$ at position $p$. Then $a \in(a b) \cdot \Lambda_{s}(X)$, thus $b \in \Lambda_{s}(X)$. Following Definition 3.2 and the remark above, if $a \in \overline{x s}$ at position $p$ then $\pi^{-1} \cdot b \in x s$ at $p$, for both translations, as stated in Lemma 3.8, resulting in $\pi \circ \pi^{-1} \cdot b \in \overline{x s^{\prime}}$ thus $b \in \overline{x s^{\prime}}$ by properties of permutations. Hence $a \in \overline{x s^{\prime}}\{a \mapsto b, b \mapsto a\}$ at $p$.

- Suppose $c=b$ such that $b \in \overline{x s}$ at position $p$. Then $b \in(a b) \cdot \Lambda_{s}(X)$, thus $a \in \Lambda_{s}(X)$. Following Definition 3.2 and the remark above, if $b \in \overline{x s}$ at position $p$ then $\pi^{-1} \cdot a \in x s$ at $p$, for both translations, as stated in Lemma 3.8, resulting in $\pi \circ \pi^{-1} \cdot a \in \overline{x s^{\prime}}$ thus $a \in \overline{x s^{\prime}}$ by properties of permutations. Hence $b \in \overline{x s^{\prime}}\{a \mapsto b, b \mapsto a\}$ at $p$.

- Suppose now $c \neq a, c \neq b$ such that $c \in \overline{x s}$ at position $p$. Then $c \in(a b) \cdot \Lambda_{s}(X)$, thus $c \in \Lambda_{s}(X)$. Following Definition 3.2 and the remark above, $\pi^{-1} \cdot c \in x s$ at $p$, for both translations, as stated in Lemma $\underline{3.8}$ resulting in $\pi \circ \pi^{-1} \cdot c \in \overline{x s^{\prime}}$ thus $c \in \overline{x s^{\prime}}$ by properties of permutations. Hence $c \in \overline{x s^{\prime}}\{a \mapsto b, b \mapsto a\}$ at $p$.

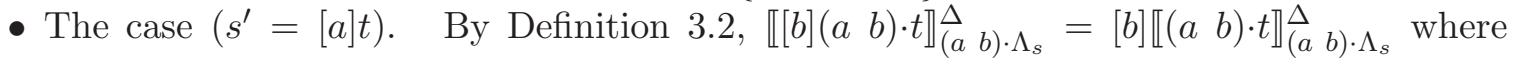
$b \in(a b) \cdot \Lambda_{s}(X)$ for any $X \in \mathcal{X}$ occurring in $t$. Take any variable $c$ not occurring free in the CRS meta-term $\llbracket\left(\begin{array}{ll}a & b\end{array}\right) \cdot t \rrbracket_{(a b) \cdot \Lambda_{s}}^{\Delta}$. Then one could choose another CRS representative $[c] \llbracket(a b) \cdot t \rrbracket_{(a b) \cdot \Lambda_{s}}^{\Delta}\{b \mapsto c\}$ in its $\alpha$-equivalence class. The induction hypothesis impliess $[c]\left(\llbracket(a b) \cdot t \rrbracket_{(a b) \cdot \Lambda_{s}}^{\Delta}\right)\{b \mapsto c\}=[c]\left(\llbracket t \rrbracket_{\Lambda_{s}}^{\Delta}\{a \mapsto b, b \mapsto a\}\right)\{b \mapsto c\}$ where $\left(\llbracket t \rrbracket_{\Lambda_{s}}^{\Delta}\{a \mapsto b, b \mapsto a\}\right)\{b \mapsto c\}$ $=\llbracket t \rrbracket_{\Lambda_{s}}^{\Delta}\{a \mapsto c\}\{b \mapsto a\}$. Since variable $c$ does not occur free in $\llbracket(a b) \cdot t \rrbracket_{(a b) \cdot \Lambda_{s}}^{\Delta}$, we can assume $[c]\left(\llbracket t \rrbracket_{\Lambda_{s}}^{\Delta}\{a \mapsto b, b \mapsto a\}\right)\{b \mapsto c\}=[c] \llbracket t \rrbracket_{\Lambda_{s}}^{\Delta}\{a \mapsto c, c \mapsto a\}\{b \mapsto a\}$ without loss of generality.

Furthermore, $\llbracket[a] t \rrbracket_{\Lambda_{s}}^{\Delta}\{a \mapsto b, b \mapsto a\}=[a]\left(\llbracket t \rrbracket_{\Lambda_{s}}^{\Delta}\{b \mapsto a\}\right)$ where $a \in \Lambda_{s}(X)$ for any $X \in \mathcal{X}$ occurring in $t$. Since there exists a variable $c$ not free in $\llbracket t \rrbracket_{\Lambda_{s}}^{\Delta}\{b \mapsto a\}$ as explained above, we choose an $\alpha$-equivalent $C R S$ meta-term $[c]\left(\llbracket t \rrbracket_{\Lambda_{s}}^{\Delta}\{a \mapsto c, c \mapsto a\}\right)\{b \mapsto a\}$ without loss of generality. And the result follows. 
The case $(s=[b] t)$ is similarly solved and thus omitted here.

- The case $\left(s^{\prime}=[c] t\right)$. By Definition [3.2, $\llbracket[c]\left(\begin{array}{ll}a & \left.b) \cdot t \rrbracket_{(a} b\right) \cdot \Lambda_{s}\end{array}=[c] \llbracket\left(\begin{array}{ll}a & b\end{array}\right) \cdot t \rrbracket_{(a b) \cdot \Lambda_{s}}^{\Delta}\right.$ where $c \in(a b) \cdot \Lambda_{s}(X)$ for any $X \in \mathcal{X}$ occurring in $t$.

Furthermore, $\llbracket[c] t \rrbracket_{\Lambda_{s}}^{\Delta}\{a \mapsto b, b \mapsto a\}=[c] \llbracket t \rrbracket_{\Lambda_{s}}^{\Delta}\{a \mapsto b, b \mapsto a\}$ where $c \in \Lambda_{s}(X)$ for any $X \in$ $\mathcal{X}$ occurring in $t$.

By induction hypothesis, $\llbracket(a b) \cdot t \rrbracket_{(a b) \cdot \Lambda_{s}}^{\Delta}=\llbracket t \rrbracket_{\Lambda_{s}}^{\Delta}\{a \mapsto b, b \mapsto a\}$ and the result follows.

- The case $\left(s^{\prime}=f t\right)$. By Definition [3.2. $\llbracket f((a b) \cdot t) \rrbracket_{(a b) \cdot \Lambda_{s}}^{\Delta}=f\left(\llbracket(a b) \cdot t \rrbracket_{(a b) \cdot \Lambda_{s}}^{\Delta}\right)$.

Furthermore, $\llbracket f t \rrbracket_{\Lambda_{s}}^{\Delta}\{a \mapsto b, b \mapsto a\}=f \llbracket t \rrbracket_{\Lambda_{s}}^{\Delta}\{a \mapsto b, b \mapsto a\}$.

By induction hypothesis, $\llbracket(a b) \cdot t \rrbracket_{(a b) \cdot \Lambda_{s}}^{\Delta}=\llbracket t \rrbracket_{\Lambda_{s}}^{\Delta}\{a \mapsto b, b \mapsto a\}$ and the result follows.

- The case $\left(s^{\prime}=\left(s_{1}, \ldots, s_{n}\right)\right)$. Note that

$$
\llbracket(a b) \cdot\left(s_{1}, \ldots, s_{n}\right) \rrbracket_{(a b) \cdot \Lambda_{s}}^{\Delta}=\llbracket\left((a b) \cdot s_{1}, \ldots,(a b) \cdot s_{n}\right) \rrbracket_{(a b) \cdot \Lambda_{s}}^{\Delta},
$$

and by Definition 3.2 ,

$$
\llbracket\left((a b) \cdot s_{1}, \ldots,(a b) \cdot s_{n}\right) \rrbracket_{(a b) \cdot \Lambda_{s}}^{\Delta}=\left(\llbracket(a b) \cdot s_{1} \rrbracket_{(a b) \cdot \Lambda_{s}}^{\Delta}, \ldots, \llbracket\left(\begin{array}{ll}
a & b
\end{array}\right) \cdot s_{n} \rrbracket_{(a b) \cdot \Lambda_{s}}^{\Delta}\right) .
$$

Furthermore,

$$
\llbracket\left(s_{1}, \ldots, s_{n}\right) \rrbracket_{\Lambda_{s}}^{\Delta}\{a \mapsto b, b \mapsto a\}=\left(\llbracket s_{1} \rrbracket_{\Lambda_{s}}^{\Delta}\{a \mapsto b, b \mapsto a\}, \ldots, \llbracket s_{n} \rrbracket_{\Lambda_{s}}^{\Delta}\{a \mapsto b, b \mapsto a\}\right) .
$$

By induction hypothesis, $\llbracket(a b) \cdot s_{i} \rrbracket_{(a b) \cdot \Lambda_{s}}^{\Delta}=\llbracket s_{i} \rrbracket_{\Lambda_{s}}^{\Delta}\{a \mapsto b, b \mapsto a\}$ where $0 \leq i \leq n$ and the result follows.

Theorem 3.13 (Uniformity w.r.t. $\alpha$ ). Let $\Delta \vdash t, \Delta \vdash s$ be a pair of closed nominal terms-in-context such that $\Delta \vdash s \approx_{\alpha} t$ and let $\hat{s}=\mathcal{T}(\Delta, s), \hat{t}=\mathcal{T}(\Delta, t)$ be their respective CRS translations according to Definition 3.2. Then $\hat{s}=\hat{t}$.

Proof. We prove a more general property:

Let $\Delta \vdash s^{\prime} \approx_{\alpha} t^{\prime}$ be a subderivation of $\Delta \vdash s \approx_{\alpha} t$. Hence, $s^{\prime}=\left.\pi \cdot s\right|_{p}$ and $t^{\prime}=\left.\rho \cdot t\right|_{p}$, for some permutations $\pi, \rho$. Then, $\llbracket s^{\prime} \rrbracket_{\Lambda_{\pi \cdot s}}^{\Delta}=\llbracket t^{\prime} \rrbracket_{\Lambda_{\rho \cdot t}}^{\Delta}$.

From this property we deduce in particular $\hat{s}=\hat{t}$ when $p=\epsilon$.

To prove this property, we proceed by induction on the derivation and distinguish cases according to the last rule used.

- $(\approx \alpha \mathbf{a})$. In this case, $s^{\prime}=a=t^{\prime}$. By Definition [3.2, $\llbracket s^{\prime} \rrbracket_{\Lambda_{\pi \cdot s}}^{\Delta}=a=\llbracket t^{\prime} \rrbracket_{\Lambda_{\rho \cdot t}}^{\Delta}$.

- $(\approx \alpha \mathbf{X})$. In this case, $s^{\prime}=\pi_{s^{\prime}} \cdot X, t^{\prime}=\rho_{t^{\prime}} \cdot X$, and $\Delta \vdash d s\left(\pi_{s^{\prime}}, \rho_{t^{\prime}}\right) \# X$. The result follows by Lemma 3.11 .

- $\left(\approx_{\alpha}[\mathbf{a}]\right)$. In this case, $s^{\prime}=[a] s^{\prime \prime}, t^{\prime}=[a] t^{\prime \prime}$, and $\Delta \vdash s^{\prime \prime} \approx_{\alpha} t^{\prime \prime}$. The result follows by induction hypothesis.

- $\left(\approx_{\alpha}[\mathbf{b}]\right)$. In this case, $s^{\prime}=[a] s^{\prime \prime}, t^{\prime}=[b] t^{\prime \prime}, \Delta \vdash(b a) \cdot s^{\prime \prime} \approx_{\alpha} t^{\prime \prime}$ and $\Delta \vdash b \# s^{\prime \prime}$.

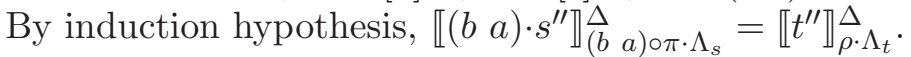

By Lemma 3.12, $\llbracket\left(\begin{array}{ll}b & a\end{array}\right) \cdot s^{\prime \prime} \rrbracket_{(b \quad a) \circ \pi \cdot \Lambda_{s}}^{\Delta}=\llbracket s^{\prime \prime} \rrbracket_{\pi \cdot \Lambda_{s}}^{\Delta}\{a \mapsto b, b \mapsto a\}$. Since $\Delta \vdash b \# s^{\prime}$ then $\llbracket s^{\prime \prime} \rrbracket_{\pi \cdot \Lambda_{s}}^{\Delta}\{a \mapsto b, b \mapsto a\}=\llbracket s^{\prime \prime} \rrbracket_{\pi \cdot \Lambda_{s}}^{\Delta}\{a \mapsto b\}$. Therefore, $\llbracket s^{\prime \prime} \rrbracket_{\pi \cdot \Lambda_{s}}^{\Delta}\{a \mapsto b\}=\llbracket t^{\prime \prime} \rrbracket_{\rho \cdot \Lambda_{t}}^{\Delta}$.

Then, $\llbracket s^{\prime} \rrbracket_{\pi \cdot \Lambda_{s}}^{\Delta}=[a] \llbracket s^{\prime \prime} \rrbracket_{\pi \cdot \Lambda_{s}}^{\Delta}=[b] \llbracket s^{\prime \prime} \rrbracket_{\pi \cdot \Lambda_{s}}^{\Delta}\{a \mapsto b\}$ since $b$ does not occur free in the translation of $s^{\prime \prime}$. And $\llbracket t^{\prime} \rrbracket_{\rho \cdot \Lambda_{t}}^{\Delta}=[b] \llbracket t^{\prime \prime} \rrbracket_{\rho \cdot \Lambda_{t}}^{\Delta}$. The result follows.

- $\left(\approx_{\alpha} \mathbf{f}\right)$. In this case, $s^{\prime}=f s^{\prime \prime}, t^{\prime}=f t^{\prime \prime}$ and $\Delta \vdash s^{\prime \prime} \approx_{\alpha} t^{\prime \prime}$. The result follows directly by induction hypothesis.

- $\left(\approx_{\alpha}\right.$ tupl $)$. In this case, $s^{\prime}=\left(s_{1}^{\prime}, \ldots, s_{n}^{\prime}\right), t^{\prime}=\left(t_{1}^{\prime}, \ldots, t_{n}^{\prime}\right)$ and $\Delta \vdash s_{1}^{\prime} \approx_{\alpha} t_{1}^{\prime}, \ldots, \Delta \vdash s_{n}^{\prime} \approx_{\alpha}$ $t_{n}^{\prime}$. The result follows directly by induction hypothesis. 


\section{Transforming NRS Rules}

NRS rules are more general than CRS rules in that unabstracted atoms may occur in rules. In this section, we impose some conditions on NRS rules to obtain a class of rules that can be translated to CRS rules.

Definition 4.1 (Standard nominal rule). A nominal rule is called standard when it is closed and the left-hand side has the form $f s$.

Definition 4.2 (Rule translation function). Let $R=\nabla \vdash l \rightarrow r$ be a standard nominal rule. The translation of $R$ is $\mathcal{T}^{\mathcal{R}}(\nabla, l, r)=\mathcal{T}(\nabla, l) \Rightarrow \mathcal{T}(\nabla, r)$, where $\mathcal{T}(\Delta, t)$ is given in Definition 3.2 .

Lemma 4.3 (Well-defined rule translation). Let $R=\nabla \vdash l \rightarrow r$ be a standard nominal rule. If $R^{\prime}=\hat{l} \Rightarrow \hat{r}$ is its translation according to Definition 4.2, then $R^{\prime}$ is a CRS rule.

Proof. First, note that if a nominal rule $\nabla \vdash l \rightarrow r$ is closed (i.e., $\nabla \vdash(l, r)$ is closed), then $\nabla \vdash l$ and $\nabla \vdash r$ are both closed terms. Hence:

- By Property 3.10 both $\hat{l}$ and $\hat{r}$ are closed CRS meta-terms.

- By definition of a nominal rule, the variables in $r$ are also in $l$. It is easy to see, by induction on Definition [3.2, that $\hat{r}$ contains only those meta-variables occurring in $\hat{l}$, and metavariables occur only in meta-applications where the arguments are lists of bound variables respecting the arity of the meta-variable (see Property [3.9). Moreover, $\llbracket l \rrbracket_{\Lambda_{l}}^{\nabla}=\llbracket l \rrbracket_{\Lambda_{(l, r)}}^{\nabla}$ and $\llbracket r \rrbracket_{\Lambda_{r}}^{\nabla}=\llbracket r \rrbracket_{\Lambda_{(l, r)}}^{\nabla}$. This is because $V(r) \subseteq V(l)$, the rule is closed and $\nabla$ is shared by all functions.

- By definition of a standard rule (see Definition 4.1), $l$ has the form $f s$.

Hence $R^{\prime}$ is a CRS rule (see Section 2.2).

Example 4.4. The (closed) nominal rules to compute prenex normal forms (see Example 2.12) can be translated to CRS rules by application of our algorithm. We show the CRS translation computed by our Haskell implementation (see [10]):

$$
\begin{array}{ll}
\text { and }(P, \text { forall }([a] Q(a))) & \Rightarrow \text { forall }([a] \text { and }(P, Q(a))) \\
\text { and }(\text { forall }([a] Q(a)), P) & \Rightarrow \text { forall }([a] \operatorname{and}(Q(a), P)) \\
\text { or }(P, \text { forall }([a] Q(a))) & \Rightarrow \text { forall }([a] \operatorname{or}(P, Q(a))) \\
\text { or }(\text { forall }([a] Q(a)), P) & \Rightarrow \text { forall }([a] \operatorname{or}(Q(a), P)) \\
\text { and }(P, \operatorname{exists}([a] Q(a))) & \Rightarrow \text { exists }([a] \text { and }(P, Q(a))) \\
\operatorname{and}(\operatorname{exists}([a] Q(a)), P) & \Rightarrow \text { exists }([a] \operatorname{and}(Q(a), P)) \\
\operatorname{or}(P, \operatorname{exists}([a] Q(a)) & \Rightarrow \operatorname{exists}([a] \operatorname{or}(P, Q(a))) \\
\operatorname{or}(\operatorname{exists}([a] Q(a)), P) & \Rightarrow \operatorname{exists}[a] \operatorname{or}(Q(a), P) \\
\operatorname{not}(\operatorname{exists}([a] Q(a))) & \Rightarrow \text { forall }([a] \operatorname{not}(Q(a))) \\
\operatorname{not}(\text { forall }([a] Q(a))) & \Rightarrow \operatorname{exists}([a] \operatorname{not}(Q(a))) .
\end{array}
$$

Note that the nominal variable $P$ becomes the CRS meta-variable $P$ of arity 0 . Hence, by definition (see [25]), if a substitute of $\mathrm{P}$ contains the free variable $a$, then the bound variable $a$ in the meta-term will be renamed to avoid name clashes. On the other hand, the nominal variable $Q$ becomes the CRS meta-variable $Q$ of arity 1, which has the bound variable $a$ as argument. 
Example 4.5. The next set of nominal rules are inspired by the simulation of $\beta$-reduction and $\eta$-reduction as defined in [15].

$\begin{array}{rlrl}(\beta \text { app }) & \operatorname{app}\left(\operatorname{lam}\left([a] \operatorname{app}\left(X, X^{\prime}\right)\right), Y\right) & \rightarrow \\ & & \left.\operatorname{app}\left(\operatorname{app}\left(\operatorname{lam}\left([a] X^{\prime}\right), Y\right), \operatorname{app}(\operatorname{lam}([a] X), Y)\right)\right), \\ \left(\beta_{\operatorname{var}}\right) & & \operatorname{app}(\operatorname{lam}([a] a), X) & \rightarrow X, \\ \left(\beta_{\Lambda}\right) & a \# Y \vdash & \operatorname{app}(\operatorname{lam}([a] Y), X) & \rightarrow Y, \\ \left(\beta_{\operatorname{lam}}\right) & b \# Y \vdash & \operatorname{app}(\operatorname{lam}([a] \operatorname{lam}([b] X)), Y) & \rightarrow \operatorname{lam}([b] \operatorname{app}(\operatorname{lam}([a] X), Y)), \\ (\eta) & a \# X \vdash & \operatorname{lam}([a] \operatorname{app}(X, a)) & \rightarrow X .\end{array}$

The CRS translation is:

$$
\begin{aligned}
& \text { ( } \beta \text { app) } \quad \operatorname{app}\left(\operatorname{lam}\left([a] \operatorname{app}\left(X(a), X^{\prime}(a)\right)\right), Y\right) \Rightarrow \\
& \left(\beta_{\Lambda}\right) \quad \operatorname{app}(\operatorname{lam}([a] Y), X) \quad \Rightarrow Y \text {, } \\
& \left(\beta_{\text {lam }}\right) \quad \operatorname{app}(\operatorname{lam}([a] \operatorname{lam}([b] X(a, b))), Y) \Rightarrow \operatorname{lam}([b] \operatorname{app}(\operatorname{lam}([a] X(a, b)), Y)) \text {, } \\
& (\eta) \operatorname{lam}([a] \operatorname{app}(X, a)) \quad \Rightarrow X \text {. }
\end{aligned}
$$

In rule $\left(\beta_{\text {lam }}\right)$, notice how both occurrences of the meta-variable $X$ share the same ordered list of bound variables, regardless of the fact that in the left-hand side, $[a]$ is above $[b]$ in the syntax tree while in the right-hand side it is the opposite. This ensures that substitutions work well, as explained in more detail in the next section.

\section{Simulating Nominal Rewrite Steps}

We consider next the relationship between the rewriting relation on nominal terms generated by a set of nominal rules $\mathcal{R}$ and the rewriting relation on CRS terms generated by its translation. Our goal is to show that the rewriting relation is preserved when nominal terms and rules are translated to CRSs.

Translation of a rewrite relation is not as straight-forward as one could expect. The rewriting relation generated by a set of CRS rules is defined on terms, not on meta-terms. Recall that CRS substitutes are terms, containing no meta-variables, preceded by the binder $\underline{\lambda}$ and a list of pairwise distinct variables (the length of the list corresponds with the arity of the meta-variable it substitutes). In order to preserve the rewriting relation, we need to consider only ground nominal substitutions. Moreover, substitutions are not translated on their own, but together with the term-in-context to be instantiated (since permutations are also applied to the substitution in order to preserve the meaning of the term). For this reason, we will define a translation function for pairs of a term-in-context $\Delta \vdash t$ and a substitution $\sigma$.

Moreover, there are swappings occurring in a permutation that can only be dealt with by applying directly the permutation to the nominal substitution before translation. These swappings correspond to mappings from atoms to unabstracted atoms occurring in the term. Dealing with these swappings at term level would contradict the property of closedness of a CRS rule. Take for instance the example

$$
(\vdash(a b) \cdot X,[X \mapsto f(a, b)]) .
$$

The term $\vdash(a b) \cdot X$ is trivially closed (no unabstracted atoms occur in the term and there is only one variable). The CRS translation given in Definition 3.2 for nominal terms and 
Definition 5.1 for substitution, given below, produce the pair

$$
(X,[X \mapsto f(b, a)])
$$

where the permutation $(a b)$ has been directly applied to the instantiation of $X, f(b, a)$ to construct the CRS substitute and not in the nominal term, since neither $a$ nor $b$ are abstracted above $X$. Further examples are considered after Definition 5.1, where we present the nominal substitution translation function.

We must ensure a nominal substitution is correctly translated with respect to the nominal term it instantiates. For this, we apply the function toAscList equally in both Definition 3.2 and Definition [5.1, over the set of mappings $\Lambda$ (see Definition [3.1), which produces a fixed and ordered list of atoms $\left[a_{1}, \ldots, a_{n}\right]$ for each nominal variable in the term. These lists are added to the substitutes for meta-variables, which have the form $\underline{\lambda}\left(a_{1}, \ldots, a_{n}\right)$.t.

Definition 5.1 (Substitution translation). Let $\Delta \vdash t$ be a closed nominal term-in-context, $\Lambda_{t}$ as in Definition [3.1, and $\sigma$ a nominal substitution satisfying $\Delta$, such that $\sigma=\left[X_{i} \mapsto\right.$ $\left.t_{i}\right], 1 \leq i \leq n$ where $\operatorname{dom}(\sigma) \subseteq V(t)$ and $t \sigma$ is ground.

Then $\mathcal{T}^{f}(\Delta, t, \sigma)=\left[X_{i} \mapsto \underline{\lambda}\left(\overline{x s}_{i}\right) \cdot s_{i}\right]$ is defined as follows:

- $\overline{x s}_{i} \triangleq \pi_{i} \cdot x s_{i}$ and,

- $x s_{i} \triangleq \operatorname{toAscList}\left(\left[\pi_{i}^{-1} \cdot \Lambda_{t}\left(X_{i}\right)\right]-\left\{a \mid a \# X_{i} \in \Delta\right\}\right)$,

- $s_{i} \triangleq \mathcal{T}\left(\Delta, \pi_{i} \cdot t_{i}\right)$ where $\pi_{i}$ is the permutation suspended in the leftmost occurrence of $X_{i}$ in $t$.

Lemma 5.4 justifies the use of the leftmost occurrence of $\pi \cdot X$ in $t$. Intuitively, each substitute generated by application of the translation function to distinct occurrences of a moderated variable is indeed $\alpha$-equivalent. Hence the leftmost occurrence is used as a representative.

We denote by $(\hat{t}, \hat{\sigma})$ the result of $\left(\mathcal{T}(\Delta, t), \mathcal{T}^{\mathcal{S}}(\Delta, t, \sigma)\right)$.

Example 5.2. Consider the following pair of a nominal term-in-context and substitution

$$
(t=\vdash f([a][b] X,[b][a] X), \sigma=[X \mapsto g(a, b)])
$$

where

$$
t \sigma=f([a][b] g(a, b),[b][a] g(a, b)) .
$$

Then, applying $\left(\mathcal{T}, \mathcal{T}^{f}\right)$ we obtain the pair

$$
(\hat{t}=f([a][b] X(a, b),[b][a] X(a, b)), \hat{\sigma}=[X \mapsto \underline{\lambda}(a \cdot b) \cdot g(a, b)]) .
$$

The CRS term $\hat{\sigma}(\hat{t})$ is computed as follows:

$$
\begin{gathered}
f([a][b](\underline{\lambda}(a, b) \cdot g(a, b))(a, b),[b][a](\underline{\lambda}(a, b) \cdot g(a, b))(a, b)) \rightarrow_{\underline{\beta}} \\
f([a][b] g(a, b),[b][a] g(a, b))
\end{gathered}
$$

which corresponds to the nominal term $t \sigma$.

Example 5.3. We revisit the nominal term

$$
\vdash[a][b](a c) \cdot X
$$


given in example 3.6, for a more detailed view on its translation to CRSs.

Assume we are given the pair

$$
(\vdash[a][b](a c) \cdot X, \sigma=[X \mapsto f(a, b, c)]) .
$$

Its CRS translation is

$$
([a][b] X(b, a), \hat{\sigma}=[X \mapsto \underline{\lambda}(b, a) \cdot f(c, b, a)])
$$

with $x s=[b, c], \overline{x s}=[b, a]$ and $(a c) \cdot \sigma(X)=f(c, b, a)$. Notice we must apply $(a c)$ to $x s$ in order to permit the capture of the variable $a$ occurring in $(a c) \cdot \sigma(X)$. Thus, the obtained meta-term mimics the behaviour of the nominal term and substitution.

Later we formally prove the application of permutations to the nominal substitution $\sigma$ does not affect the uniqueness of $\hat{\sigma}$ modulo $\alpha$, when more than one occurrence of the same meta-variable appears in the term. Intuitively, if there are more occurrences of $X$, Property 3.10 implies the initial list of bindings we call $x s$ is syntactically equivalent for all occurrences of $X$ thus it binds the same atoms. Considering $\pi$ is applied to both $x s$ and $X \sigma$, renamings do not affect the structure of the binding. Moreover, for any other renaming of atoms $a \mapsto \pi(a)$ occurring during application of $\pi \cdot \sigma(X)$, an identical mapping must exist in all other occurrences of $\pi \cdot X$ else it contradicts the property of closedness (see Definition 2.14) therefore $a \# X \in \Delta$.

For instance, consider the following pair of a closed term-in-context and a substitution.

$$
(a, c \# X \vdash g([a][b][c](a d)(e f) \cdot X,[a][b][c](c d)(e f) \cdot X),[X \mapsto f(b, d, e, f)]) .
$$

The term translation function produces a CRS meta-term

$$
g([a][b][c] X(b, a),[a][b][c] X(b, c))
$$

and the substitution translation produces the corresponding substitute

$$
[X \mapsto \underline{\lambda}(b, a) . f(b, a, f, e)] .
$$

Note that, if translation of the substitution is done with respect to each variable in the term, we obtain:

$[X \mapsto \underline{\lambda}(b, a) . f(b, a, f, e)]$ and $[X \mapsto \underline{\lambda}(b, c) . f(b, c, f, e)]$ for each occurrence of $X$. These substitutes are indeed $\alpha$-equivalent and our algorithm outputs the leftmost $[X \mapsto \underline{\lambda}(b, a) . f(b, a, f, e)]$.

Moreover, applying the lexical ordering directly to $\overline{x s}$ instead of $x s$ would produce substitutes which are no longer $\alpha$-equivalent, providing incorrect instantiations.

We now formalise the property of equivalence modulo $\alpha$ of substitution occurrences after translation. The intuition is they are equivalent because of terms being closed (see Definition 2.14) and sharing an initially equivalent variable binding list, xs (see Property 3.8).

Lemma 5.4 ( $\alpha$-equivalence of substitutes). Let $\Delta \vdash t$ be a closed nominal term-in-context, $\Lambda_{t}$ as defined in Definition 3.1, and $\sigma$ a nominal substitution satisfying $\Delta$ such that $\operatorname{dom}(\sigma) \subseteq$ $V(t)$ and $t \sigma$ is ground. Let $\pi_{i} \cdot X, \pi_{j} \cdot X$ be two occurrences of the same variable in $t$, and let $\left[X \mapsto \underline{\lambda}\left(\overline{x s}_{i}\right) . s_{i}\right]$ and $\left[X \mapsto \underline{\lambda}\left(\overline{x s}_{j}\right) . s_{j}\right]$ be translations according to Definition $[5.1$ but using $\pi_{i}$ and $\pi_{j}$ respectively. Then $\left[X \mapsto \underline{\lambda}\left(\overline{x s}_{i}\right) . s_{i}\right] \approx_{\alpha}\left[X \mapsto \underline{\lambda}\left(\overline{x s}_{j}\right) \cdot s_{j}\right]$.

Proof. By definition, $\mathcal{T}^{f}\left(\Delta, t,\left[X_{i} \mapsto t_{i}\right]\right)=\left[X_{i} \mapsto \underline{\lambda}\left(\overline{x s}_{i}\right) . s_{i}\right]$ with $\overline{x s}_{i} \triangleq \pi_{i} \cdot x s_{i}, x s_{i} \triangleq$ toAscList $\left(\left[\pi_{i}^{-1} \cdot \Lambda_{t}\left(X_{i}\right)\right]-\left\{a \mid a \# X_{i} \in \Delta\right\}\right)$ and $s_{i} \triangleq \mathcal{T}\left(\Delta, \pi_{i} \cdot t_{i}\right)$ where $\pi_{i} \cdot X_{i}$ is the leftmost occurrence of $X_{i}$. 
Hence, each atom $a \in \operatorname{support}\left(\pi_{i}\right)$ with $\pi_{i}(a) \notin \Lambda_{t}(X)$ must satisfy $\pi_{i}(a)=\pi_{j}(a)$, so that when $\pi_{i}, \pi_{j}$ are applied to each occurrence of $t_{i}$ during translation, they remain equivalent, therefore $s_{i}=s_{j}$. Otherwise $a \in d s\left(\pi_{i}, \pi_{j}\right)$ such that $a \# X \in \Delta$ by Definition 2.14. Since $\sigma$ must also satisfy $\Delta$, it is the case that $a \# t_{i}$, and application of either $\pi_{i}$ or $\pi_{j}$ to $t_{i}$ produces no changes.

Now we look at the binding list added to the substitute (i.e., $\overline{x s}_{i}, \overline{x s}_{j}$ ). Property 3.8 states that $x s$ is shared by all occurrences, and the term is closed, then it is the case that each variable in $x s$ binds the same variable in $t_{i}$ for both occurrences. It immediately follows that by application of $\pi_{i}, \pi_{j}$ to each occurrence of $x s$ and $t_{i}$, the renaming of bound variables does not affect the binding structure, hence they are $\alpha$-equivalent. Finally, for any other atom $a \in \Lambda_{t}(X)$ but $a \notin \overline{x s}$ it can only be that $\pi^{-1}(a) \# X \in \Delta$ hence $\pi^{-1}(a) \notin x s$. It also does not occur free in $t_{i}$ since it must satisfy $\Delta$, as previously stated. Therefore it does not alter the outcome of the translation.

This shows that the choice of the leftmost element in the translation does not affect correctness.

We are now ready to prove that substitutions are correctly translated.

Lemma 5.5 (Instantiation). Let $\Delta \vdash t$ be a closed nominal term-in-context, $\Lambda_{t}$ as defined in Definition 3.1, and $\sigma$ a substitution satisfying $\Delta$ such that $\operatorname{dom}(\sigma) \subseteq V(t)$ and $t \sigma$ is ground.

Suppose $\left(\llbracket t^{\prime} \rrbracket_{\Lambda_{t}}^{\Delta}, \mathcal{T}^{f}(\Delta, t, \sigma)\right)=\left(\hat{t}^{\prime}, \hat{\sigma}\right)$, for any subterm $t^{\prime}$ of $t\left(\right.$ e.g. $\left.t^{\prime}=t\right)$, is a recursive call in the translation process.

Then $\llbracket t^{\prime} \sigma \rrbracket_{\Lambda_{t}}^{\Delta}=\hat{\sigma}\left(\hat{t}^{\prime}\right)$.

Proof. By induction on the structure of $t^{\prime}$.

(atom): If $t^{\prime}=a$, the property holds trivially.

(var): If $t^{\prime}=\pi \cdot X$, then we distinguish cases with respect to $\Lambda_{t}(X)$.

(1) If $\Lambda_{t}(X)=\varnothing$, then it immediately follows that $\left(\llbracket \pi \cdot X \rrbracket_{\varnothing}^{\Delta}, \mathcal{T}^{\mathcal{S}}(\Delta, t, \sigma)\right)=(X, \hat{\sigma})$ where $\hat{\sigma}(X)=s \triangleq \mathcal{T}(\Delta, \pi \cdot \sigma(X))$ by definition of substitution translation. Therefore $\hat{\sigma}(X)=s$. This is equivalent to $\llbracket \pi \cdot X \sigma \rrbracket_{\varnothing}^{\Delta}=s$.

(2) If $\Lambda_{t}(X) \neq \varnothing$, then $\left(\llbracket \pi \cdot X \rrbracket_{\Lambda_{t}}^{\Delta}, \mathcal{T}^{f}(\Delta, t, \sigma)\right)=(X(\overline{x s}), \hat{\sigma}(X)=\underline{\lambda}(\overline{x s}) \cdot s)$, where $s=$ $\mathcal{T}(\Delta, \pi \cdot \sigma(X))$. Therefore $(X(\overline{x s})) \hat{\sigma}(X)=(\underline{\lambda}(\overline{x s}) \cdot s)(\overline{x s})=s$. This is also equivalent to $\llbracket(\pi \cdot X) \sigma(X) \rrbracket_{\Lambda_{t}}^{\Delta}=s$.

(abs): If $t^{\prime}=[a] s$, then $\llbracket([a] s) \sigma \rrbracket_{\Lambda_{t}}^{\Delta}=\llbracket[a] s \sigma \rrbracket_{\Lambda_{t}}^{\Delta}=[a] \llbracket s \sigma \rrbracket_{\Lambda_{t}}^{\Delta}=[a] \hat{\sigma}(\hat{s})=\hat{\sigma}([a] \hat{s})$ where $\hat{\sigma}(\hat{s})=\llbracket s \sigma \rrbracket_{\Lambda_{t}}^{\Delta}$ by the induction hypothesis.

(fun): If $t^{\prime}=f s$, then $\llbracket(f s) \sigma \rrbracket_{\Lambda_{t}}^{\Delta}=\llbracket f(s \sigma) \rrbracket_{\Lambda_{t}}^{\Delta}=f \llbracket(s \sigma) \rrbracket_{\Lambda_{t}}^{\Delta}=f(\hat{\sigma}(\hat{s}))=\hat{\sigma}(f \hat{s})$ where $\hat{\sigma}(\hat{s})=\llbracket s \sigma \rrbracket_{\Lambda_{t}}^{\Delta}$ by the induction hypothesis.

(tuple): If $t^{\prime}=\left(s_{1}, \ldots, s_{n}\right)$, then $\llbracket\left(s_{1}, \ldots, s_{n}\right) \sigma \rrbracket_{\Lambda_{t}}^{\Delta}=\llbracket\left(\left.s_{1} \sigma\right|_{V\left(s_{1}\right)}, \ldots,\left.s_{n} \sigma\right|_{V\left(s_{n}\right)}\right) \rrbracket_{\Lambda_{t}}^{\Delta}=$ $\left(\hat{\sigma}_{1}\left(\hat{s}_{1}\right), \ldots, \hat{\sigma}_{n}\left(\hat{s_{n}}\right)\right)$ where each $\hat{\sigma}_{i}\left(\hat{s}_{i}\right)=\left.\llbracket s_{i} \sigma\right|_{V\left(s_{i}\right)} \rrbracket_{\Lambda_{t}}^{\Delta}$ by the induction hypothesis. And $\left.\hat{\sigma}\left(\hat{s}_{i}\right)\right|_{V\left(s_{i}\right)}=\hat{\sigma}_{i}\left(\hat{s}_{i}\right)$, as a consequence of Lemma 5.4

Nominal variable translation depends both on freshness context and abstractions occurring above the variable. The translation function uses them to build both the list of arguments of a meta-application and the list of binders added to a substitute, whereas the syntaxdirected nature of the translating function transforms the rest of the elements directly. By keeping track of the abstractions above a variable via function $\Lambda$, translating any subterm $t^{\prime}$ 
of $t$ with $\Lambda_{t}(X)$ results in the same term as translating $t^{\prime}$ within $t$, as stated in the following lemma.

Note that $C[$ ] is a term, as explained in the paragraph above Definition [2.13, and is translated to $\hat{C}[]$ using Definition 3.2 .

Lemma 5.6 (Context). Let $\Delta \vdash t$ be a nominal term-in-context such that $t=C[s]$ (i.e., $t=C[-\mapsto s])$, where $s$ is a ground nominal term. Assume $\llbracket C \rrbracket_{\Lambda_{C}}^{\Delta}=\hat{C}$ and $\llbracket s \rrbracket_{\varnothing}^{\Delta}=\hat{s}$. Then $\llbracket C[s] \rrbracket_{\Lambda_{t}}^{\Delta}=\hat{C}[\hat{s}]$.

Proof. This is a particular case of Lemma [5.5, where $\sigma=[-\mapsto s]$. If $\mathcal{T}^{f}(\Delta, t, \sigma)=\hat{\sigma}$ then $\llbracket C[s] \rrbracket_{\Lambda_{t}}^{\Delta}=\hat{C}[\hat{s}]$ since $s$ is ground, hence $\Lambda_{C}=\Lambda_{t}$.

We can now derive the main result of the paper: the preservation of the rewrite relation under the translation.

Theorem 5.7 (Preservation of reduction). Let $R=\nabla \vdash l \rightarrow r$ be a standard nominal rule. Let $t$ be a ground nominal term and $\hat{t}=\mathcal{T}(\varnothing, t)$. If $t \rightarrow_{R} u$ then $\hat{t} \Rightarrow_{R^{\prime}} \hat{u}$ using $R^{\prime}=\mathcal{T}^{\mathcal{R}}(\nabla, l, r)$, and $\hat{u}=\mathcal{T}(\varnothing, u)$.

Proof. If $t \rightarrow_{R} u$ then there exists $C, \sigma$ such that $t \approx_{\alpha} C[l \sigma]$ with $\sigma$ a ground nominal substitution satisfying $\nabla$ such that $\operatorname{dom}(\sigma) \subseteq V(l)$.

Also $R^{\prime}=\mathcal{T}^{\mathcal{R}}(\nabla, l, r)=\llbracket l \rrbracket_{\Lambda_{l}}^{\nabla} \Rightarrow \llbracket r \rrbracket_{\Lambda_{r}}^{\nabla}=\hat{l} \Rightarrow \hat{r}$ by Definition 4.2, where Lemma 4.3 asserts that the translation is a CRS rule.

If we have, by application of Definition [5.1, $\mathcal{T}^{f}(\nabla, l, \sigma)=\hat{\sigma}_{l}$ then, by Lemma 5.5] $\llbracket l \sigma \rrbracket_{\Lambda_{l \sigma}}^{\nabla}=\hat{\sigma}_{l}(\hat{l})$. Hence we have $\hat{t}=\hat{C}\left[\hat{\sigma}_{l}(\hat{l})\right]$ by Lemmas 5.6 and Property 3.13 .

Similarly, since $u \approx_{\alpha} C[r \sigma]$ we have $\mathcal{T}^{\mathcal{S}}(\nabla, r, \sigma)=\hat{\sigma}_{r}$, leading to $\hat{u}=\hat{C}\left[\hat{\sigma}_{r}(\hat{r})\right]$ by application of Definition 5.1, followed by Lemmas 5.5, 5.6 and Property 3.13. Notice that $\operatorname{dom}\left(\hat{\sigma}_{r}\right) \subseteq \operatorname{dom}\left(\hat{\sigma}_{l}\right)$ and $\hat{\sigma}_{r}(\hat{X}) \approx_{\alpha} \hat{\sigma}_{l}(\hat{X})$ by Lemma 5.4 .

Hence we conclude by stating that if $l \sigma \rightarrow_{R} r \sigma$ then $\hat{t} \Rightarrow_{R^{\prime}} \hat{u}$ as expected.

Corollary 5.8 (Termination). Termination of the translated CRS implies termination of the NRS.

\section{Translating from CRSs to NRSs: An Improved Approach}

Our goal is to obtain a tool capable of translating rules back and forth between CRSs and NRSs. Based on the CRS to NRS translation defined in [16], we now provide an improved algorithm to translate CRS rules to closed nominal rules.

Since CRSs follow Barendregt's naming convention (each abstraction uses a different bound variable to avoid name clashes), we will work with closed rewriting (see Definition 2.15), where in each rewriting step a freshened copy of the rule is chosen, making the translation easier.

6.1. Translating Meta-terms in CRS Rewrite Rules. We begin by defining a pair of auxiliary functions.

Function $\Phi$ provides the leftmost meta-application for each meta-variable occurring in the left-hand side of a CRS rule $l$. More precisely, $\Phi_{l}\left(Z_{i}^{n}\right)=\left[a_{1}, \ldots, a_{n}\right]$ if $Z_{i}^{n}\left(a_{1}, \ldots, a_{n}\right)$ is the leftmost occurrence of $Z_{i}^{n}$ in $l$. We use it in the translation to ensure the preservation of both closedness (see Lemmas 6.13 \& 6.14) and the rewriting relation (see Theorem 6.25). 
The second auxiliary function, $\Psi$, is used to convert each meta-application $Z_{i}^{n}\left(a_{1}, \ldots, a_{n}\right)$ occurring in the left-hand side $l$ of a CRS rule into a list $\pi_{i}$ of swappings, such that $\pi_{i} \cdot Z_{i}$, when instantiated, simulates the $\beta$-reduction of a valuation $\sigma$ applied to each occurrence of $Z_{i}^{n}$. To accomplish this, $\Psi$ is parameterised by $\Phi_{l}\left(Z_{i}^{n}\right)$, and applied locally to each argument list $\left(b_{1}, \ldots, b_{n}\right)$ in a meta-application of $Z_{i}^{n}$ which is not the leftmost one, in order to preserve $\alpha$-equality along the NRS translation.

Both auxiliary functions will be used when defining the translation algorithm for leftand right-hand sides of CRS rules (Definitions 6.5 \& 6.10). We start by providing a formal definition of $\Phi$.

Definition 6.1. Given a closed CRS meta-term $t$, the partial mapping $\Phi_{t}$ from metavariables to lists of variables is defined such that

$$
\Phi_{t}\left(Z_{i}^{n}\right)=\left[a_{1}, \ldots, a_{n}\right]
$$

if the leftmost occurrence of the meta-variable $Z_{i}^{n}$ in $t$ has the form $Z_{i}^{n}\left(a_{1}, \ldots, a_{n}\right)$, where $a_{1}, \ldots, a_{n}$ are pairwise distinct bound variables. We denote by $\Phi_{t}\left(Z_{i}^{n}\right)_{k}$ the $k$ th element in the list $\Phi_{t}\left(Z_{i}^{n}\right)$.

To provide a behaviour similar to that of CRSs, NRSs must maintain the relation among argument lists occurring for a meta-variable $Z_{i}^{n}$ along a CRS meta-term. This relation is one of position within the argument list such that all (possibly distinct) variables at a position $k$ (where $1 \leq k \leq n$ ), for each argument list adjacent to an occurrence of $Z_{i}^{n}$, are $\beta$-reduced by application of binder $\underline{\lambda} x_{k}$ in a substitute for a valuation $\sigma\left(Z_{i}^{n}\right)$. Furthermore, consider a non left-linear closed meta-term (i.e., more than one occurrence of a meta-variable exists) of the form $\left([a] Z_{i}^{n}(a),[b] Z_{i}^{n}(b)\right)$. By the property of $\alpha$-equivalence in CRSs, $[a] Z_{i}^{n}(a)$ and $[b] Z_{i}^{n}(b)$ are considered syntactically equal. A direct translation of this meta-term to the NRS term $([a] Z,[b] Z)$ is not suitable: $[a] Z$ and $[b] Z$ are not $\alpha$-equivalent and, moreover, this is not a closed NRS term. Both translation issues are approached by the same methodology: making use of the NRSs tools to check $\alpha$-equivalence of terms, that is, swappings and the freshness relation.

To construct a list of swappings for an occurrence of a non-leftmost NRS variable $Z$ with respect to both $\Phi_{t}\left(Z_{i}^{n}\right)$ and a variable argument list $\left(b_{1}, \ldots, b_{n}\right)$ adjacent to that same CRS occurrence $Z_{i}^{n}$, we need to convert a permutation in two-line notation into a series of $k$-cycles where $k \geq 2$ (Id permutations are discarded) followed by a decomposition into swappings, as explained in Example 2.32. Notice that the set of variables in $\Phi_{t}\left(Z_{i}^{n}\right)$ and $\left(b_{1}, \ldots, b_{n}\right)$ can be equivalent, disjoint or sharing some variables, therefore it is not a bijection per se. However, the variables in each list are distinct among them and the length of the list is the same in both cases, so there is a one-to-one correspondence between each variable in $\Phi_{t}\left(Z_{i}^{n}\right)$ and $\left(b_{1}, \ldots, b_{n}\right)$. This is enough to define a cycle notation (therefore forcing the construction of bijections).

Example 6.2. Consider the closed CRS meta-term

$$
t=[c]([a][b] Z(a, b, c),[x][y] Z(x, c, y))
$$

where variable name $c$ is shared between meta-applications. Application of $\Phi$ to $t$ generates $\Phi_{t}(Z)=[a, b, c]$ and, to convert the set of mappings $\{a \rightarrow x, b \rightarrow c, c \rightarrow y\}$, going from the leftmost argument list to the rightmost one, into a list of swappings, we transform the implicit two-line notation (variables in the domain of the mapping on the top row and their respective image on the bottom row) into the cycle notation $(a, x)(b, c, y)$ and finally into 
the list of swappings $\pi_{Z}=\left(\begin{array}{ll}y & b\end{array}\right)\left(\begin{array}{lll}c & b\end{array}\right)\left(\begin{array}{ll}x & a\end{array}\right)$ as informally described in Section 2.3. Then, $\pi_{Z}(a)=x, \pi_{Z}(b)=c, \pi_{Z}(c)=y$ as expected. Also, $\pi_{Z}(x)=a$ and $\pi_{Z}(y)=c$, however both variables $x, y$ do not belong to the domain $\Phi_{t}(Z)$ of the mappings therefore their image is irrelevant for a correct translation of the permutation list. Moreover, it will be shown in the translation (Definition 6.5) that both atoms must be fresh for the nominal variable $Z$, i.e., $x \# Z, y \# Z \in \Delta$ to preserve closedness of the NRS translation. Hence one could say that auxiliary function $\Psi$ upgrades directed paths (i.e., finite sequence of edges connecting distinct vertices without repetition) to cycles.

Next is the formal definition of $\Psi$.

When constructing cycles, the main difficulty originates when a variable chosen by $\Psi$ as the initial element of the sequence is part of a directed path. As a result, there may be elements preceding in the sequence which are not reachable by constructing the sequence rightwards from the initial element. The solution: construct the sequence leftwards from the initial element only in the case $\Psi$ is not dealing with a real cycle (thus we must know whether $\Psi$ is dealing with a cycle or a directed path at each recursive step). Here is how.

Definition 6.3. Let $s=\left[a_{1}, \ldots, a_{n}\right]$ and $t=\left[b_{1}, \ldots, b_{n}\right]$ be any two pairs of lists of length $n$ over the set $\mathcal{V}$ of variables, and $f:\left[a_{1}, \ldots, a_{n}\right] \mapsto\left[b_{1}, \ldots, b_{n}\right], f^{-1}:\left[b_{1}, \ldots, b_{n}\right] \mapsto\left[a_{1}, \ldots, a_{n}\right]$ a pair of mappings such that $b_{k}=f\left(a_{k}\right)$ and $a_{k}=f^{-1}\left(b_{k}\right)$, for $1 \leq k \leq n$. Then, $\Psi(s, t)$ returns a list $\pi$ of swappings over the set $\mathcal{A}$ of atoms, recursively defined as follows:

$$
\begin{aligned}
\Psi(\text { nil }, \text { nil }) & = \\
\Psi\left(\left[a_{1}, \ldots, a_{n}\right],\left[b_{1}, \ldots, b_{n}\right]\right)= & \left(a_{m} b_{k}\right)\left(a_{m} b_{j}\right) \cdots\left(a_{m} b_{1}\right)\left(a_{m} a_{1}\right)\left(a_{m} a_{i}\right) \cdots\left(a_{m} a_{l}\right) \\
& \circ \Psi\left(s_{1}, t_{1}\right) \quad \text { where } 1 \leq i, j, k, l, m \leq n \text { and }
\end{aligned}
$$

- $\left(\begin{array}{ll}a_{m} & b_{k}\end{array}\right)\left(\begin{array}{ll}a_{m} & b_{j}\end{array}\right) \cdots\left(\begin{array}{ll}a_{m} & b_{1}\end{array}\right)\left(\begin{array}{ll}a_{m} & a_{1}\end{array}\right)\left(\begin{array}{ll}a_{m} & a_{i}\end{array}\right) \cdots\left(\begin{array}{ll}a_{m} & a_{l}\end{array}\right)$ is the 2-cycle decomposition (see Property 2.31) of the permutation in cycle form $C=\left(a_{m}, a_{l}, \ldots, a_{i}, a_{1}, b_{1}, \ldots, b_{j}, b_{k}\right)$;

- $C$ is constructed by successive applications of functions $f$ and $f^{-1}$ over $a_{1}$ (as many times as possible) as follows:

$$
a_{m} \stackrel{f^{-1}\left(a_{l}\right)}{\longleftrightarrow} a_{l} \cdots \stackrel{f^{-1}\left(a_{i}\right)}{\longleftrightarrow} a_{i} \stackrel{f^{-1}\left(a_{1}\right)}{\longleftarrow} a_{1} \stackrel{f\left(a_{1}\right)}{\longmapsto} b_{1} \stackrel{f\left(b_{1}\right)}{\longmapsto} \cdots b_{j} \stackrel{f\left(b_{j}\right)}{\longmapsto} b_{k}
$$

where $f^{-1}\left(a_{1}\right)$ and $f\left(b_{j}\right)$ are only applicable when $b_{k} \neq a_{1}$. Otherwise, if $b_{k}=a_{1}$ then the cycle form would be $\left(a_{1}, b_{1}, \ldots, b_{j}\right)$, generating then a list of swappings $\left(a_{1} b_{j}\right) \cdots\left(a_{1} b_{1}\right)$.

- $s_{1}=s \backslash C$,

- $t_{1}=t \backslash C$.

Example 6.4. Using the definition on the ordered pair of variable lists $[a, b, c],[x, c, y]$ from the above example results in mappings:

$$
f(a)=x \quad f(b)=c \quad f(c)=y \quad f^{-1}(x)=a \quad f^{-1}(c)=b \quad f^{-1}(y)=c
$$

Then, function $\Psi$ begins by applying $f$ to variable $a$ such that

$$
a \stackrel{f(a)}{\longmapsto} x
$$

and since $x \notin \operatorname{domain}(f), a \notin \operatorname{domain}\left(f^{-1}\right)$ :

$$
\Psi([a, b, c],[x, c, y])=(a x) \circ \Psi([b, c],[c, y])
$$

where lists $[b, c],[c, y]$ are obtained by elimination of variables $a, x$ from both lists. Finally,

$$
\Psi([b, c],[c, y])=(b y)(b c) \circ \mathrm{Id}
$$


is created as follows. Starting with variable $b$,

$$
b \stackrel{f(b)}{\longmapsto} c \stackrel{f(c)}{\longmapsto} y
$$

where $y \notin \operatorname{domain}(f)$ and $b \notin \operatorname{domain}\left(f^{-1}\right)$ and thus the cycle is $(b, c, y)$, converting to $\left(\begin{array}{ll}b & y\end{array}\right)\left(\begin{array}{ll}b & c\end{array}\right)$. Also, $\Psi(n i l, n i l)=\mathrm{Id}$.

Notice the positioning of swapping $(a x)$ is different from the previous example solution. Previously we had $\pi_{Z}=(y b)(c b)(x a)$ whereas, by application of Definition 6.3, it resolves to $(x a)(y b)(c b)$ instead (Id is omitted). However, Lemma 2.29 stated that disjoint cycles commute therefore both permutations perform equivalent actions.

To translate a CRS rule $l \Rightarrow r$, two different functions, called Left and Right, are applied to $l$ and $r$ respectively, both parameterised by $\Phi_{l}$. We define them separately.

The translation function Left uses an auxiliary function working on pairs: $(\Delta, l)_{\Phi_{l}}^{\Lambda}$, such that Left $(l)=(\varnothing, l)_{\Phi_{l}}^{\varnothing}$ where $l$ is a meta-term and $\Delta, \Lambda$ are recursively constructed. $\Delta$ has freshness constraints to avoid certain names appearing, in order to keep the nominal term consistently named throughout, since there are no naming conventions in NRSs. $\Lambda$ is a set of variables such that the recursive call $\left(\Delta, Z_{i}^{n}\left(a_{1}, \ldots, a_{n}\right)\right)_{\Phi_{l}}^{\Lambda}$ has in $\Lambda$ those variables bound above $Z_{i}^{n}$.

For any occurrence of a meta-variable $Z_{i}^{n}$ in $l$ that is not the leftmost, $\Lambda \backslash \Phi_{l}\left(Z_{i}^{n}\right)$ contains the set of atoms that must be fresh for $Z_{i}$ in the NRS translation to translate consistently from CRSs to NRSs and back again. That is, $\Lambda \backslash \Phi_{l}\left(Z_{i}^{n}\right)$ is the set of all variables bound above $Z_{i}^{n}$ that cannot occur in the translated NRS term, otherwise the NRS term is no longer closed (see Definition 2.14). We provide examples after the formal definition.

Definition 6.5 (Left translation). Let $s$ be a closed CRS meta-term where all the metaapplications have the form $Z\left(a_{1}, \ldots, a_{n}\right)$ such that $a_{1}, \ldots, a_{n}$ are different bound variables. Let $\Phi$ be the function given in Definition 6.1 and $\Psi$ the function in Definition 6.3. Then $\operatorname{Left}(s)=(\varnothing, s)_{\Phi_{s}}^{\varnothing}$, where $(\Delta, s)_{\Phi_{s}}^{\Lambda}$ is inductively defined as follows:

$$
\begin{aligned}
(\Delta, a)_{\Phi_{s}}^{\Lambda}= & (\Delta, a) \\
(\Delta,[a] t)_{\Phi_{s}}^{\Lambda}= & \left(\Delta^{\prime},[a] t^{\prime}\right), \\
& \text { where }\left(\Delta^{\prime}, t^{\prime}\right)=(\Delta, t)_{\Phi_{s}}^{\Lambda \cup\{a\}} \\
= & \left(\Delta^{\prime}, f t^{\prime}\right), \\
& \text { where }\left(\Delta^{\prime}, t^{\prime}\right)=(\Delta, t)_{\Phi_{s}}^{\Lambda} \\
(\Delta, f t)_{\Phi_{s}}^{\Lambda}= & \left(\Delta^{\prime},\left(t_{1}^{\prime}, \ldots, t_{n}^{\prime}\right)\right), \\
& \text { where }\left(\Delta, t_{k}\right)_{\Phi_{s}}^{\Lambda}=\left(\Delta_{k}, t_{k}^{\prime}\right), \text { for } 1 \leq k \leq n \\
\left(\Delta,\left(t_{1}, \ldots, t_{n}\right)\right)_{\Phi_{s}}^{\Lambda}= & \text { and } \Delta^{\prime}=\bigcup_{k} \Delta_{k} \\
\left(\Delta, Z_{i}^{n}\left(a_{1}, \ldots, a_{n}\right)\right)_{\Phi_{s}}^{\Lambda}= & \left(\Delta \cup \Delta^{\prime}, Z_{i}\right) \text { if leftmost occurrence of } Z_{i}^{n} \text { in } s \\
& \text { where } \Delta^{\prime}=\left\{a \# Z_{i} \mid a \in \Lambda \backslash \Phi_{s}\left(Z_{i}^{n}\right)\right\} \\
\left(\Delta, Z_{i}^{n}\left(b_{1}, \ldots, b_{n}\right)\right)_{\Phi_{s}}^{\Lambda}= & \left(\Delta \cup \Delta^{\prime}, \Psi\left(\Phi_{s}\left(Z_{i}^{n}\right),\left[b_{1}, \ldots, b_{n}\right]\right) \cdot Z_{i}\right) \text { otherwise, } \\
& \text { where } \Delta^{\prime}=\left\{b \# Z_{i} \mid b \in \Lambda \backslash \Phi_{s}\left(Z_{i}^{n}\right)\right\}
\end{aligned}
$$

Therefore, the left-hand side translation function does the following: for each meta-variable $Z_{i}^{n}$ in $s$, if $Z_{i}^{n}$ is the leftmost subterm of the form $Z_{i}^{n}\left(a_{1}, \ldots, a_{n}\right)$, it is replaced by $Z_{i}$ and for 
each $a \in \Lambda$ but not in $Z_{i}^{n}\left(a_{1}, \ldots, a_{n}\right), a \# Z_{i}$ is added to $\Delta$, whereas the rest of the subterms with the form $Z_{i}^{n}\left(b_{1}, \ldots, b_{n}\right)$ are replaced by $\Psi\left(\Phi_{s}\left(Z_{i}^{n}\right),\left[b_{1}, \ldots, b_{n}\right]\right) \cdot Z_{i}$. Additionally, $b_{j} \# Z_{i}$ is added to $\Delta$ for each $b_{j} \in \Lambda \backslash \Phi_{s}\left(Z_{i}^{n}\right), 1 \leq j \leq n$, that is, for any binder with variables distinct to the binders in the leftmost occurrence, a freshness constraint is added in the translated term to preserve closedness. No further freshness constraints are needed since we are working with closed nominal rewriting.

For the examples in this and next sections, $X, Y, Z, \ldots$ range over the meta-variables instead of $Z_{i}^{n}$, without loss of generality. This notation is closer to nominal rewriting notation and we also find it more readable. We write $Z_{i}^{n}$ when providing definitions to follow the standard CRS notation.

The following lemma is similar to Lemma 3.3, but we are now translating from CRS meta-terms to NRS terms.

Lemma 6.6 (Preservation of meta-variables as variables in the Left translation). Suppose $t$ is a CRS meta-term and $\left(\Delta, t^{\prime}\right)=$ Left $(t)$ its translation by Definition 6.5. Then, $Z_{i}^{n}\left(a_{1}, \ldots, a_{n}\right)$ is an occurrence of a meta-application in $t$ with $Z_{i}^{n}$ at position $p$ if and only if $\pi \cdot Z_{i}$ occurs in $t^{\prime}$ at position $p$.

Proof. By induction over the structure of $t$ and the fact that there is a one-to-one correspondence between the elements of $t$ and $t^{\prime}$ as it is shown in the syntax-directed translation function in Definition 6.10. Therefore, a meta-variable $Z_{i}^{n}$ occurs at position $p$ in $t$, along with a list of variable arguments $\left(a_{1}, \ldots, a_{n}\right)$ (when $n>0$ ), if and only if there exists a variable $\pi \cdot Z_{i}$ at $p$ in its translation $t^{\prime}$.

Lemma 6.7 (Preservation of free variables as unabstracted atoms in the Left translation). Let $l$ be a closed CRS meta-term, $\Phi_{l}$ as defined in Definition 6.1 and $\left(\nabla, l^{\prime}\right)=\operatorname{Left}(l)$ as in Definition 6.5. Assume $(\Delta, s)_{\Phi_{l}}^{\Lambda}=\left(\Delta^{\prime}, s^{\prime}\right)$ is computed in the translation of $l$, where $s$ is any subterm of $l$ (e.g. $s=l)$. Then, $a$ is free in $s$ if and only if $a$ is an unabstracted atom in $s^{\prime}$. Hence, there are no unabstracted atom subterms in $l^{\prime}$, since $l$ is closed.

Proof. By induction on the definition of Left.

(var): If $s=a$ then $(\Delta, a)_{\Phi_{l}}^{\Lambda}=(\Delta, a)$ and the property holds. Note that, since $l$ is closed, $s$ is a strict subterm of $l$ and there exists an abstraction for $a$ in $l$ and above $s$. Therefore $[a]$ occurs above $a$ in the translated term too, by the syntax-directed nature of the translation function.

(Mvar): If $s=Z_{i}^{n} t$, then $\left(\Delta, Z_{i}^{n} t\right)_{\Phi_{l}}^{\Lambda}=\left(\Delta^{\prime}, \pi \cdot Z_{i}\right)$ and the property holds trivially.

(abs): If $s=[a] t$ then $(\Delta,[a] t)_{\Phi_{l}}^{\Lambda}=\left(\Delta^{\prime},[a] t^{\prime}\right)$ where $\left(\Delta^{\prime}, t^{\prime}\right)=(\Delta, t)_{\Phi_{l}}^{\Lambda \cup\{a\}}$ and the property holds for $(\Delta, t)_{\Phi_{l}}^{\Lambda \cup\{a\}}$ by induction hypothesis.

(fun): If $s=f t$ then $(\Delta, f t)_{\Phi_{l}}^{\Lambda}=\left(\Delta^{\prime}, f t^{\prime}\right)$ where $\left(\Delta^{\prime}, t^{\prime}\right)=(\Delta, t)_{\Phi_{l}}^{\Lambda \cup\{a\}}$ and the property holds for $(\Delta, t)_{\Phi_{l}}^{\Lambda \cup\{a\}}$ by induction hypothesis.

(tuple): If $s=\left(t_{1}, \ldots, t_{n}\right)$ then $\left(\Delta,\left(t_{1}, \ldots, t_{n}\right)\right)_{\Phi_{l}}^{\Lambda}=\left(\Delta^{\prime},\left(t_{1}^{\prime}, \ldots, t_{n}^{\prime}\right)\right)$ where each $\left(\Delta_{i}^{\prime}, t_{i}^{\prime}\right)=$ $\left(\Delta, t_{i}\right)_{\Phi_{l}}^{\Lambda}, \Delta^{\prime}=\bigcup \Delta_{i}^{\prime}$ for $1 \leq i \leq n$ and the property holds for each $\left(\Delta, t_{i}\right)_{\Phi_{l}}^{\Lambda}$ by induction hypothesis.

The other direction is similar.

Example 6.8. The CRS meta-term $f([a] X,[b] X)$ is translated using Left as the closed nominal term

$$
a \# X, b \# X \vdash f([a] X,[b] X) \text {. }
$$


Thanks to the use of closed nominal rewriting, less freshness constraints are needed in the translation than when using (standard) nominal rewriting. However, in some cases freshness constraints are generated, even if a translation without freshnesses might be possible. For example, the CRS meta-term $f([a] X(a),[b] X(b))$ produces the closed nominal translation

$$
b \# X \vdash f([a] X,[b](a b) \cdot X)
$$

where $b \# X$ ensures that the term is closed (see Definition $[3.2)$; however, $\vdash f([a] X,[a] X)$ would also be a correct translation (note that $b \# X \vdash f([a] X,[b](a b) \cdot X) \approx_{\alpha} f([a] X,[a] X)$ ).

The translation function $\operatorname{Right}(\cdot)$ for the right-hand side of a CRS rule, when applied to a closed meta-term $r$, produces $\left(\Delta_{r}, \llbracket r \rrbracket_{\Phi_{l}}\right)$, where subterms of the form $Z_{i}^{n}\left(t_{1}, \ldots, t_{n}\right)$ in $r$ are translated as terms of the form $\left(\pi \cdot Z_{i}^{n}\right)\left[\Phi_{r}\left(Z_{i}^{n}\right)_{k} \mapsto \llbracket t_{k} \rrbracket_{\Phi_{l}}\right]$ such that, for the cases where $t_{k} \in\left(t_{1}, \ldots, t_{n}\right)$ is not a variable in $r$, the translation to NRS introduces a notation for explicit atom substitution by means of an extra set of rules (see Definition 6.9) added to the given system. These explicit substitution rules are handled separately from the specified rules in the NRS, namely they are discarded if translating back into CRSs by application of the function defined in Section 4.

On the other hand, a swapping $\left(\Phi_{l}\left(Z_{i}^{n}\right)_{k} t_{k}\right)$ is added to $\pi$ where $t_{k}$ is a variable occurring in $r$ (i.e. $t_{k}$ is a variable occurring bound above $Z_{i}^{n}$ by definition of a closed CRS meta-term). Also $\Delta_{r}$ contains fresh atoms for each bound variable occurring in the term.

We remark that function $\Psi$ is not needed here. CRSs are assumed to follow the usual naming conventions, that is, different bound variables are used in each abstraction. Hence, there are no clashes among variable names both in $\Phi_{l}\left(Z_{i}^{n}\right)$ and other occurrences of $Z_{i}^{n}$ in the right-hand side meta-term.

We use the notation for explicit atom substitution given in [16, where $t[a \mapsto s]$ is an abbreviation for $\operatorname{sub}([a] t, s)$. We recall below the rules, then continue by formalising the definition of the right-hand side rule translation.

Definition 6.9 (Explicit substitution rules). The following (closed) nominal rewrite rules define the behaviour of the binary function symbol sub. The notation $t[a \mapsto s]$ is syntactic sugar for $\operatorname{sub}([a] t, s)$

$$
\begin{aligned}
& \left(\sigma_{\text {var }}\right) \quad \vdash a[a \mapsto X] \quad \rightarrow X \\
& \left(\sigma_{\epsilon}\right) \quad a \# Y \vdash \quad Y[a \mapsto X] \quad \rightarrow Y \\
& \left(\sigma_{f}\right) \quad \vdash(f X)[a \mapsto Y] \quad \rightarrow f X[a \mapsto Y] \text { for each } f \text { in } \Sigma \\
& \left(\sigma_{\text {prod }}\right) \quad \vdash\left(X_{1}, \ldots, X_{n}\right)[a \mapsto Y] \rightarrow\left(X_{1}[a \mapsto Y], \ldots, X_{n}[a \mapsto Y]\right) \\
& \left.\left(\sigma_{a b s}\right) \quad b \# Y \vdash([b] X)[a \mapsto Y] \quad \rightarrow \quad b\right](X[a \mapsto Y])
\end{aligned}
$$

Definition 6.10 (Right translation). Let $l \Rightarrow r$ be a CRS rule. Let $\Phi_{l}$ be the function defined in Definition 6.1 applied to the CRS meta-term $l$. Then $\operatorname{Right}(r)=\left(\Delta_{r}, \llbracket r \rrbracket_{\Phi_{l}}\right)$ where

$$
\Delta_{r}=\left\{a_{k} \# Z_{i}^{n} \mid a_{k} \text { occurs bound above } Z_{i}^{n} \text { in } r\right\}
$$


and $\llbracket r \rrbracket_{\Phi_{l}}$ is defined by:

$$
\begin{array}{ll}
\llbracket a \rrbracket \rrbracket_{\Phi_{l}}= & a \\
\llbracket & f \llbracket s \rrbracket_{\Phi_{l}} \\
\llbracket f s \rrbracket_{\Phi_{l}} & {\left[a \rrbracket \llbracket s \rrbracket_{\Phi_{l}}\right.} \\
\llbracket[a] s \rrbracket_{\Phi_{l}} & \\
\llbracket\left(t_{1}, \ldots, t_{n}\right) \rrbracket_{\Phi_{l}=}= & \left(\llbracket t_{1} \rrbracket_{\Phi_{l}}, \ldots, \llbracket t_{n} \rrbracket_{\Phi_{l}}\right) \\
\llbracket Z_{i}^{n} t \rrbracket_{\Phi_{l}}= & \pi \cdot Z_{i} \text { with } \pi=\text { Id if } n=0, \text { otherwise } \\
& n>0, t=\left(t_{1}, \ldots, t_{n}\right) \text { such that } \\
& \left(\pi \cdot Z_{i}\right)\left[\Phi_{l}\left(Z_{i}^{n}\right)_{m 1} \mapsto \llbracket t_{m 1} \rrbracket_{\Phi_{l}}\right] \ldots\left[\Phi_{l}\left(Z_{i}^{n}\right)_{m k} \mapsto \llbracket t_{m k} \rrbracket_{\Phi_{l}}\right] \text { where } \\
& \pi=\left(\Phi_{l}\left(Z_{i}^{n}\right)_{j 1} \llbracket t_{j 1} \rrbracket \Phi_{l}\right) \ldots \cdot\left(\Phi_{l}\left(Z_{i}^{n}\right)_{j k} \llbracket t_{j k} \rrbracket \Phi_{l}\right), \text { and } \\
& j 1 \ldots j k, m 1 \ldots m k \in\{1, \ldots, n\}, \\
& \llbracket t_{j 1} \rrbracket_{\Phi_{l}}, \ldots, \llbracket t_{j k} \rrbracket_{\Phi_{l}} \in \mathcal{A}
\end{array}
$$

Similar to Definition 6.5, we take into account abstracted variables which do not appear in the meta-application.

Note that, compared with the translation given in [16], we generate less freshness constraints and have also improved the flow when translating back and forth between CRS and NRS rules, namely by converting explicit substitution among atoms into swappings added to $\pi$ (see Example 6.17 and further examples in Section 7 ).

The following pair of lemmas state that function Right also preserves meta-variables as variables and free variables as unabstracted atoms during the translation of a CRS metaterm.

Lemma 6.11 (Preservation of meta-variables as variables in the Right translation). Suppose $t$ is a CRS meta-term and $\left(\Delta, t^{\prime}\right)=$ Right $(t)$ its translation by Definition 6.10 . Then, $Z_{i}^{n}\left(t_{1}, \ldots, t_{n}\right)$ is an occurrence of a meta-application in $t$ with $Z_{i}^{n}$ at position $p$ if and only if $\pi \cdot Z_{i}$ occurs in $t^{\prime}$ at position $p$.

Proof. By induction over the structure of $t$. The proof is similar to the proof in Lemma 6.6 and thus omitted.

Lemma 6.12 (Preservation of free variables as unabstracted atoms in the Right translation). Let $l, r$ be a pair of closed CRS meta-terms, $\Phi_{l}$ as defined in Definition 6.1 and $\left(\Delta, r^{\prime}\right)=$ Right $(r)$ as in Definition 6.10. Assume $\llbracket s \rrbracket_{\Phi_{l}}=s^{\prime}$ is computed in the translation of $r$, where $s$ is any subterm of $r(e . g . s=r)$. Then, $a$ is free in $s$ if and only if $a$ is an unabstracted atom in $s^{\prime}$. Hence, there are no unabstracted atom subterms in $r^{\prime}$, since $r$ is closed.

Proof. By induction on the definition of Right. The interesting case is that of a metavariable since the rest of the cases are solved similarly to the proof given in Lemma 6.7 (and thus omitted here).

(Mvar): If $s=Z_{i}^{n} t$, then $\llbracket Z_{i}^{n} t \rrbracket_{\Phi_{l}}=\pi \cdot Z_{i}\left[b_{k} \mapsto t_{k}^{\prime}\right]$ where $t_{k}^{\prime}=\llbracket t_{k} \rrbracket_{\Phi_{l}}$, for some $b_{k}=\phi_{l}\left(Z_{i}^{n}\right)_{k}$ and $\left.t_{k} \in t\right|_{p} \wedge t_{k} \notin \mathcal{V}$ where $p$ is a position in $t$. The property holds by induction hypothesis on $t_{k}^{\prime}$.

Next, we prove that Definitions 6.5 and 6.10 produce closed nominal terms, separately, and also as part of the translation of a CRS rule.

Lemma 6.13. Let $t$ be the left-hand side of a CRS rule following Barendregt's naming convention (i.e., variable names are pairwise distinct), and $(\varnothing, t)_{\Phi_{t}}^{\varnothing}=\left(\Delta, t^{\prime}\right)$ its translation as in Definition 6.5, where $\Phi$ is the function in Definition 6.1. Then $\Delta \vdash t^{\prime}$ is a closed nominal term-in-context. 
Proof. For $\Delta \vdash t^{\prime}$ to be a closed term-in-context (see Definition 2.14) we must prove the following:

(1) no unabstracted atoms occur in $t^{\prime}$,

(2) any pair $\pi_{a} \cdot Z_{i}, \pi_{b} \cdot Z_{i}$ occurring in $t^{\prime}$ satisfy conditions $2 \& 3$ in Definition 2.14

Condition (1) holds because, by definition, CRS rules are closed and the translation does not introduce new atoms as proved in Lemma 6.7.

For condition (2), assume $Z_{i}^{n}\left(a_{1}, \ldots, a_{n}\right), Z_{i}^{n}\left(b_{1}, \ldots, b_{n}\right)$ are two occurrences of a metaapplication for the same meta-variable in $t$ along with their respective list of distinct variables (possibly empty, thus $Z_{i}^{0}$ ), then their translation results in $\pi_{a} \cdot Z_{i}, \pi_{b} \cdot Z_{i}$ (Lemma 6.6). We must consider three possible cases: the case where the list of atoms is empty, $(a)$, the case where one of the occurrences is the leftmost, $(b)$, and finally the case where none of the occurrences is the leftmost, $(c)$. No other cases are possible.

(a) If the list of atoms is empty (thus $\left.Z_{i}^{0}\right)$, both $\operatorname{support}\left(\pi_{a}\right), \operatorname{support}\left(\pi_{b}\right)=\varnothing$ in $\pi_{a} \cdot Z_{i}$, $\pi_{b} \cdot Z_{i}$ by definition of the translation function where for each variable $a \in \Lambda$ in the recursive call $\left(\Delta, Z_{i}^{0}\right)_{\Phi_{t}}^{\Lambda}$ (i.e., any abstraction occurring above the meta-variable), $a \# Z_{i}$ is added to $\Delta$. Hence the property of closedness holds for this case.

(b) If one of the occurrences is the leftmost in $t$, for instance $Z_{i}^{n}\left(a_{1}, \ldots, a_{n}\right)$, then its translation is $\pi_{a} \cdot Z_{i}$ with $\operatorname{support}\left(\pi_{a}\right)=\varnothing$ and $\Phi_{t}\left(Z_{i}^{n}\right)=\left[a_{1}, \ldots, a_{n}\right]$ where $\left(a_{1}, \ldots, a_{n}\right) \# Z_{i} \notin$ $\Delta$. On the other hand, $Z_{i}^{n}\left(b_{1}, \ldots, b_{n}\right)$ is translated as $\pi_{b} \cdot Z$ with $\Psi\left(\Phi_{t}\left(Z_{i}^{n}\right),\left[b_{1}, \ldots, b_{n}\right]\right)=$ $\pi_{b}$ and $b_{k} \# Z_{i} \in \Delta$ such that $b_{k} \in \Lambda \backslash \Phi_{t}\left(Z_{i}^{n}\right)$.

This means that $\left(a_{1}, \ldots, a_{n}\right)$ may appear unabstracted in an instance of $Z_{i}$ but not any of $\left\{b_{1}, \ldots, b_{n}\right\}$ distinct from $\left\{a_{1}, \ldots, a_{n}\right\}$. If so, $\left(a_{1}, \ldots, a_{n}\right)$ are all abstracted above $\pi_{a} \cdot Z_{i}$ since the meta-term is closed and $\left(\pi_{b}\left(a_{1}\right)=b_{1}, \ldots, \pi_{b}\left(a_{n}\right)=b_{n}\right)$ thus $\left(a_{1}, \ldots, a_{n}\right)$ are also abstracted above $\pi_{b} \cdot Z_{i}$. For any other atom that may appear in an instance of $Z_{i}$, the atom is unabstracted above both occurrences. Hence the property also holds for this case.

(c) If none of these occurrences is leftmost, let $Z_{i}^{n}\left(c_{1}, \ldots, c_{n}\right)$ be the leftmost one. Now $\Phi_{t}\left(Z_{i}^{n}\right)=\left[c_{1}, \ldots, c_{n}\right]$, and the translation of both occurrences in question is $\pi_{a} \cdot Z_{i}$ with $\left\{c_{1}, \ldots, c_{n}, a_{1}, \ldots, a_{n}\right\} \in \operatorname{support}\left(\pi_{a}\right)$ and $\pi_{b} \cdot Z_{i}$ with $\left\{c_{1}, \ldots, c_{n}, b_{1}, \ldots, b_{n}\right\} \in \operatorname{support}\left(\pi_{b}\right)$ where $a_{k}, b_{k} \# Z_{i} \in \Delta$ for any $a_{k} \in \Lambda_{a} \backslash \Phi_{t}\left(Z_{i}^{n}\right)$ and $b_{k} \in \Lambda_{b} \backslash \Phi_{t}\left(Z_{i}^{n}\right)$ with $\Lambda_{a}, \Lambda_{b}$ the set of variables bound over meta-applications $Z_{i}^{n}\left(a_{1}, \ldots, a_{n}\right)$ and $Z_{i}^{n}\left(b_{1}, \ldots, b_{n}\right)$, respectively. This means that any of $\left\{c_{1}, \ldots, c_{n}\right\}$ may appear unabstracted in an instance of $Z_{i}$ but not any of $\left\{a_{1}, \ldots, a_{n}, b_{1}, \ldots, b_{n}\right\}$ distinct from $\left\{c_{1}, \ldots, c_{n}\right\}$. If so, $\left(\pi_{a}\left(c_{1}\right)=\right.$ $\left.a_{1}, \ldots, \pi_{a}\left(c_{n}\right)=a_{n}\right)$ and $\left(\pi_{b}\left(c_{1}\right)=b_{1}, \ldots, \pi_{b}\left(c_{n}\right)=b_{n}\right)$ by Definition 6.3, therefore $\left(c_{1}, \ldots, c_{n}\right)$ are abstracted above both $\pi_{a} \cdot Z_{i}, \pi_{b} \cdot Z_{i}$ respectively. For any other atom that may appear in an instance of $Z_{i}$, the atom is unabstracted above both occurrences. Hence $\Delta \vdash t^{\prime}$ is a closed nominal term-in-context as expected.

Lemma 6.14. Let $t$ be the right-hand side meta-term of a CRS rule following Barendregt's naming convention, $\Phi$ the function defined in Definition 6.1 and for each meta-variable $Z_{i}^{n}$ in $t, Z_{i}^{n} \in \operatorname{dom}\left(\Phi_{s}\right)$ for the left-hand side meta-term s such that $\Phi_{s}\left(Z_{i}^{n}\right)=\left[a_{1}, \ldots, a_{n}\right]$.

Let Right $(t)=\left(\Delta_{t}, \llbracket t \rrbracket_{\Phi_{s}}\right)$ be the translation as in Definition 6.10, then $\Delta_{t} \vdash \llbracket t \rrbracket_{\Phi_{s}}$ is a closed term-in-context.

Proof. Similarly to Lemma 6.13. for $\Delta_{t} \vdash \llbracket t \rrbracket_{\Phi_{s}}$ to be a closed term-in-context we must prove that no atom subterm occurs unabstracted in $\llbracket t \rrbracket_{\Phi_{s}}$. This is the case due to CRS rules being closed by definition (see Definition [6.16) and Lemma 6.12 stating that no unabstracted 
atoms are introduced in the term by the translation function $\llbracket \cdot \rrbracket_{\Phi_{s}}$. Moreover, it must also be proved that any $\pi \cdot Z_{i}$ occurring in $t^{\prime}$ satisfy conditions $2 \& 3$ in Definition 2.14. We have already proved this case in Lemma 6.13 where none of the meta-variable occurrences is the leftmost (case $(c)$ in the proof). Function $\llbracket \cdot \rrbracket_{\Phi_{s}}$ treats each meta-variable occurrence as a non-leftmost one and there exists a leftmost meta-application $Z_{i}^{n}\left(a_{1}, \ldots, a_{n}\right)$ in $s$. Then, the result follows using Lemma 6.11 and the fact that $\Delta_{t}$ contains a freshness constraint, $b \# X$, for all atoms $b$ abstracted in $t^{\prime}$ and all variables $X \in V\left(t^{\prime}\right)$ (see Definition 6.10).

In the right-hand side of a rule, there is the possibility of explicit substitutions of form $r^{\prime}\left[a_{k} \mapsto u^{\prime}\right]$ appearing in the translated nominal term where $a_{k} \in \Phi_{s}\left(Z_{i}^{n}\right)$. Notice that $r^{\prime}\left[a_{k} \mapsto u^{\prime}\right]$ is syntactic sugar for $\operatorname{sub}\left(\left[a_{k}\right] r^{\prime}, u^{\prime}\right)$ where $r, u$ are subterms of the closed meta-term $t$. Then, any free variable in $r, u$ is in the scope of an abstraction in $t$ and, by Lemma 6.12, the same happens in their translations $r^{\prime}=\llbracket r \rrbracket_{\Phi_{s}}$ and $u^{\prime}=\llbracket u \rrbracket_{\Phi_{s}}$

Remark 6.15 (CRS term translation). For any CRS term $t, t$ is also a nominal ground term, trivially, since there are no meta-variables.

6.2. Transforming CRS Rules. In this section we show how CRS rules are converted into closed NRS rules.

Definition 6.16. We define the translation of the CRS rule $l \Rightarrow r$ as $\mathcal{C}^{\mathcal{R}}(l, r)=\Delta \vdash l^{\prime} \rightarrow r^{\prime}$, where Left $(l)=\left(\Delta_{l}, l^{\prime}\right)$, Right $(r)=\left(\Delta_{r}, r^{\prime}\right)$ and $\Delta=\Delta_{l} \cup \Delta_{r}$.

We give some examples to illustrate the definition.

Example 6.17. The translation of the $\beta$-rule shown in Example 2.21 according to Definition 6.16 is

$$
\vdash \operatorname{app}\left(\operatorname{lam}([a] Z), Z^{\prime}\right) \rightarrow Z\left[a \mapsto Z^{\prime}\right] .
$$

Now, consider the CRS differentiation operator rule as taken from [16]:

$$
\operatorname{diff}([a] \sin (Z(a))) \Rightarrow[b] \operatorname{mult}(\operatorname{app}(\operatorname{diff}([c] Z(c)), b), \cos (Z(b))) .
$$

The translation of this rule is

$$
b \# Z, c \# Z \vdash \operatorname{diff}([a] \sin (Z)) \rightarrow[b] \operatorname{mult}(\operatorname{app}(\operatorname{diff}([c](a c) \cdot Z), b), \cos ((a b) \cdot Z))
$$

where the freshness conditions are needed to preserve closedness, and mappings of atoms on the right-hand side are transformed into permutations.

Further examples can be found in Section 7

Lemma 6.18. Let $R=l \Rightarrow r$ be a CRS rule. If $\Delta \vdash l^{\prime} \rightarrow r^{\prime}$ is its translation according to Definition [6.16, then $\Delta \vdash l^{\prime} \rightarrow r^{\prime}$ is a closed nominal rewrite rule.

Proof. Consequence of Lemmas 6.13 and 6.14, and the fact that $\Phi_{l}$ is shared by the translation functions for both sides of the rule. 
Next, we proceed by providing a mechanism to translate valuations to nominal substitutions, that is, to convert each CRS substitute into a ground nominal term. Recall that we are assuming each binder binds a different variable (Barendregt's convention), and that CRS terms coincide with nominal ground terms (Remark 6.15).

The intuition behind the definition of the translation for a valuation, which is used to instantiate a CRS rule, is to rename bound variables from the list of binders, $\underline{\lambda}(\cdots)$, in a substitute for $Z_{i}^{n}$ to match those in the list of arguments for the leftmost meta-application of $Z_{i}^{n}$ in the left-hand side of the rule.

Definition 6.19 (Valuation translation). Let $t$ be a closed CRS meta-term, $\Phi_{t}$ as in Definition 6.1, and $\sigma$ a safe valuation such that $\sigma=\left[Z_{i}^{n} \mapsto \underline{\lambda}\left(a_{1}, \ldots, a_{n}\right) \cdot s_{i}\right]$ for $1 \leq i \leq m$ where $\operatorname{dom}(\sigma) \subseteq M V(t)$ and $\sigma(t)$ is ground. Then, $\langle\sigma\rangle_{\Phi_{t}} \triangleq\left[Z_{i} \mapsto \pi_{i} \cdot s_{i}\right]$ where $\pi_{i}=$ $\left(a_{n} \Phi_{t}\left(Z_{i}^{n}\right)_{n}\right) \cdots\left(a_{1} \Phi_{t}\left(Z_{i}^{n}\right)_{1}\right)$.

Below we denote by $\left(\nabla \vdash t^{\prime}, \sigma^{\prime}\right)$ the result of $\left(\operatorname{Left}(t),\langle\sigma\rangle_{\Phi_{t}}\right)$.

Example 6.20. We revisit the closed CRS meta-term $t=[c]([a][b] Z(a, b, c),[x][y] Z(x, c, y))$ given in Example 6.2, adding a safe valuation $\sigma=[Z \mapsto \underline{\lambda}(d, e, f) . g(d, e, f, z)]$. where

$$
\sigma(t)=[c]([a][b] g(a, b, c, z),[x][y] g(x, c, y, z)) .
$$

Then,

$$
\left(\operatorname{Left}(t),\langle\sigma\rangle_{\Phi_{t}}\right)=\left(x \# Z, y \# Z \vdash[c]([a][b] Z,[x][y](y b)(c b)(x a) \cdot Z), \sigma^{\prime}=[Z \mapsto g(a, b, c, z)]\right)
$$

where $g(a, b, c, z)$ was obtained by applying $(f c)(e b)(d a)$ to $g(d, e, f, z)$.

Since $\sigma^{\prime}$ satisfies the freshness constraints (i.e., $\varnothing \vdash x \# \sigma^{\prime}(Z), y \# \sigma^{\prime}(Z)$ ), the instantiation resolves to

$$
t^{\prime} \sigma^{\prime}=[c]([a][b] g(a, b, c, d),[x][y] g(x, c, y, d))
$$

which corresponds with the CRS term $\sigma(t)$.

A proof of correctness for the translation of valuations follows. Since there are two distinct functions to translate the left and right hand side of rules, we must also provide a proof for each.

Lemma 6.21 states the correctness property for the left-hand side instantiation. Suppose $\left(\operatorname{Left}(t),\langle\sigma\rangle_{\Phi_{t}}\right)=\left(\nabla \vdash t^{\prime}, \sigma^{\prime}\right)$. The proof of correctness is more involved than the instantiation proof in Lemma 5.5, due to the presence of the freshness context $\nabla$. It is not sufficient to prove that $\sigma^{\prime}$ correctly instantiates $t^{\prime}$, we must also verify that $\sigma^{\prime}$ satisfies the constraints in $\nabla$. This is the case since the valuation $\sigma$ is safe with respect to the CRS metaterm $t$ by Definition 2.25, hence free variables in $\sigma$ cannot occur in $t$, and the function $\langle\cdot\rangle_{\Phi}$ renames bound variables in $\sigma\left(Z_{i}^{n}\right)$ to coincide with the list of variables $\left(a_{1}, \ldots, a_{n}\right)$ in the leftmost occurrence of a meta-application for each meta-variable $Z_{i}^{n}$ in $t$. Recall that Left creates freshness constraints for all abstractions in a meta-term, except for the abstractions whose variables occur in the list of arguments for the leftmost meta-application of each $Z_{i}^{n}$.

Lemma 6.21 (Left instantiation). Let l be the left-hand side of a CRS rule (hence, a closed CRS meta-term); $\nabla, \Delta, \Delta^{\prime}$ freshness contexts and $\Phi$ the function given in Definition [6.1. Assume Left $(l)=\nabla \vdash l^{\prime}$ according to Definition 6.5, and let $\sigma$ be a safe valuation such that $\operatorname{dom}(\sigma) \subseteq M V(l)$ and $\sigma(l)$ is a CRS term.

Suppose $\left((\Delta, s)_{\Phi_{l}}^{\Lambda},\langle\sigma\rangle_{\Phi_{l}}\right)=\left(\left(\Delta^{\prime}, s^{\prime}\right), \sigma^{\prime}\right)$, for any subterm $s$ of $l(e . g ., s=l)$, is a recursive call in the translation process.

Then, $(\varnothing, \sigma(s))_{\Phi_{l}}^{\Lambda}=\left(\varnothing, s^{\prime} \sigma^{\prime}\right)$ where $\sigma^{\prime}$ satisfies $\Delta^{\prime}$, that is, $\vdash \Delta^{\prime} \sigma^{\prime}$. 
Proof. By induction on the structure of $s$ and the fact that $\nabla \vdash l^{\prime}$ is a closed term-in-context by Lemma 6.13 .

(var): If $s=a$, the property holds trivially.

(Mvar): If $s=Z_{i}^{n} t$, there are three cases to consider: $n=0, n>0$ and $Z_{i}^{n} t$ is the leftmost occurrence of the meta-variable $Z_{i}^{n}$ in $l$, and $n>0$ and $Z_{i}^{n} t$ is not the leftmost occurrence of $Z_{i}^{n}$ in $l$. No other cases are possible. We assume, without loss of generality, $\sigma=$ $\left[Z_{i}^{n} \mapsto \underline{\lambda}\left(a_{1}, \ldots, a_{n}\right) . s_{i}\right]$ with $s_{i}$ a CRS term for all $i$, where distinct variables $a_{1}, \ldots, a_{n}$ are bound in $s_{i}$. Then,

(1) In the case where $n=0$, that is, the meta-variable has arity 0 , it is a fact that $t=()$ and also $\sigma=\left[Z_{i}^{0} \mapsto s_{i}\right]$. Therefore, $\left(\varnothing, \sigma\left(Z_{i}^{0}\right)\right)_{\Phi_{l}}^{\Lambda}=\left(\varnothing, s_{i}\right)$ by Remark 6.15, Now, $\left(\left(\Delta, Z_{i}^{0}\right)_{\Phi_{l}}^{\Lambda},\langle\sigma\rangle_{\Phi_{l}}\right)$ produces a pair, where the substitution is $\sigma^{\prime}=\left[Z_{i} \mapsto \pi_{i} \cdot s_{i}\right]$, and $\Delta^{\prime}$ contains constraints of the form $a \# Z$ (possibly empty) for each $a \in \Lambda$ (i.e., for any abstraction occurring above $s)$, and $\pi_{i}=\operatorname{Id}$ since $t=\left(a_{1}, \ldots, a_{n}\right)=()$. Then, $Z_{i} \sigma^{\prime}=s_{i}$.

Finally, for each $a \# Z \in \Delta^{\prime}, \varnothing \vdash a \# s_{i}$ as a result of the safety conditions for rewriting in CRSs stated in Definition 2.25, which, in the nominal framework translates to: $\operatorname{supp}\left(s_{i}\right) \neq A\left(\Delta^{\prime} \vdash l^{\prime}\right)$. Hence $\sigma^{\prime}$ satisfies $\Delta^{\prime}$, i.e., $\vdash \Delta^{\prime} \sigma^{\prime}$ as required.

(2) In the case where $n>0$ and $Z_{i}^{n} t$ is the leftmost occurrence of this metavariable in $l$, we proceed as follows: since the meta-variable has arity $n, t=\left(b_{1}, \ldots, b_{n}\right)$, where the variables $b_{j}, 1 \leq j \leq n$, are all different, and also different from the variables in $\left(a_{1}, \ldots, a_{n}\right)$, since we are using Barendregt's convention. Then,

$\left(\varnothing, \sigma\left(Z_{i}^{n} t\right)\right)_{\Phi_{l}}^{\Lambda}=\left(\varnothing,\left(\underline{\lambda}\left(a_{1}, \ldots, a_{n}\right) \cdot s_{i}\right)\left(b_{1}, \ldots, b_{n}\right) \Rightarrow_{\beta} s_{i}\left[a_{1} \mapsto b_{1}, \ldots, a_{n} \mapsto b_{n}\right]\right)$.

Note that

$$
\left(\left(\Delta, Z_{i}^{n}\left(b_{1}, \ldots, b_{n}\right)\right)_{\Phi_{l}}^{\Lambda},\langle\sigma\rangle_{\Phi_{l}}\right)=\left(\left(\Delta^{\prime}, Z_{i}\right), \sigma^{\prime}=\left[Z_{i} \mapsto \pi_{i} \cdot s_{i}\right]\right),
$$

where $\Delta^{\prime}$ contains constraints of the form $a \# Z$ (possibly empty) for each $a \in \Lambda \backslash$ $\left\{b_{1}, \ldots, b_{n}\right\}$, and $\pi_{i}=\left(a_{n} b_{n}\right) \cdots\left(a_{1} b_{1}\right)$. Then, $Z_{i} \sigma^{\prime}=\pi_{i} \cdot s_{i}$ where $b_{1}, \ldots, b_{n} \notin A\left(s_{i}\right)$ following the convention.

Finally, for each $a \# Z \in \Delta^{\prime}, \vdash a \# \pi_{i} \cdot s_{i}$ as a result of the safety conditions for rewriting in CRSs (see Definition 2.25); note that $a \notin\left\{b_{1}, \ldots, b_{n}\right\}$ since this tuple is the leftmost argument list in $l$ for $Z_{i}^{n}$. Hence $\sigma^{\prime}$ satisfies $\Delta^{\prime}$, i.e., $\vdash \Delta^{\prime} \sigma^{\prime}$ as required.

(3) In the case where $n>0$ and $Z_{i}^{n} t$ is not the leftmost occurrence, the proof is similar to the previous case: $t=\left(b_{1}, \ldots, b_{n}\right)$, where the variables $b_{j}, 1 \leq j \leq n$ are all different and also different from the variables in $\left(a_{1}, \ldots, a_{n}\right)$. Then,

$\left(\Delta, \sigma\left(Z_{i}^{n} t\right)\right)_{\Phi_{l}}^{\Lambda}=\left(\varnothing,\left(\underline{\lambda}\left(a_{1}, \ldots, a_{n}\right) . s_{i}\right)\left(b_{1}, \ldots, b_{n}\right) \Rightarrow_{\beta} s_{i}\left[a_{1} \mapsto b_{1}, \ldots, a_{n} \mapsto b_{n}\right]\right)$.

Also, we have

$$
\left(\left(\Delta, Z_{i}^{n}\left(b_{1}, \ldots, b_{n}\right)\right)_{\Phi_{l}}^{\Lambda},\langle\sigma\rangle_{\Phi_{l}}\right)=\left(\left(\Delta^{\prime} \sigma^{\prime}, \pi^{\prime} \cdot Z_{i}\right), \sigma^{\prime}=\left[Z_{i} \mapsto \pi_{i} \cdot s_{i}\right]\right),
$$

where $\Delta^{\prime}$ contains constraints of the form $a \# Z$ (possibly empty) for all $a \in \Lambda \backslash \Phi_{l}\left(Z_{i}^{n}\right)$; $\pi_{i}=\left(a_{n} \Phi_{l}\left(Z_{i}^{n}\right)_{n}\right) \cdots\left(a_{1} \Phi_{l}\left(Z_{i}^{n}\right)_{1}\right)$ and $\pi^{\prime} \operatorname{maps} \pi^{\prime}\left(\Phi_{l}\left(Z_{i}^{n}\right)_{1}\right)=b_{1}, \ldots, \pi^{\prime}\left(\Phi_{l}\left(Z_{i}^{n}\right)_{n}\right)=$ $b_{n}$ by Definition $6.3(\Psi)$. Then, $\left(\pi^{\prime} \cdot Z_{i}\right) \sigma^{\prime}=\pi^{\prime} \cdot\left(\pi_{i} \cdot s_{i}\right)=\left(\pi^{\prime} \circ \pi_{i}\right) \cdot s_{i}$, by application of Definition 2.4, such that $\left(\pi^{\prime} \circ \pi_{i}\right)\left(a_{1}\right)=b_{1}, \ldots,\left(\pi^{\prime} \circ \pi_{i}\right)\left(a_{n}\right)=b_{n}$ where $\Phi_{l}\left(Z_{i}^{n}\right)_{1}, \ldots \Phi_{l}\left(Z_{i}^{n}\right)_{n}, b_{1}, \ldots, b_{n} \notin A\left(s_{i}\right)$ by convention. 
To conclude, for each $b_{i} \in\left\{b_{1}, \ldots, b_{n}\right\} \backslash \Lambda, b_{i} \# Z \in \Delta^{\prime}$, and $\varnothing \vdash b_{i} \# \pi_{i} \cdot s_{i}$ as a result of the safety conditions for rewriting in CRSs stated in Definition 2.25, thus it is a fact that $b_{i}$ does not range over $\left\{a_{1}, \ldots, a_{n}\right\} \cup \Lambda$. Hence $\sigma^{\prime}$ satisfies $\Delta^{\prime}$, as required. Then, the result follows.

(abs): If $s=[a] t$, then $(\Delta,[a] t)_{\Phi_{l}}^{\Lambda}=\left(\Delta^{\prime},[a] t^{\prime}\right)$, where $(\Delta, t)_{\Phi_{l}}^{\Lambda \cup\{a\}}=\left(\Delta^{\prime}, t^{\prime}\right)$.

By the induction hypothesis, $(\varnothing, \sigma(t))_{\Phi_{l}}^{\Lambda \cup\{a\}}=\left(\varnothing, t^{\prime} \sigma^{\prime}\right)$. Also by induction, $\vdash \Delta^{\prime} \sigma^{\prime}$. The result follows, since $\left([a] t^{\prime}\right) \sigma^{\prime}=[a] t^{\prime} \sigma^{\prime}$.

(fun): If $s=f t$, then $(\Delta, f t)_{\Phi_{l}}^{\Lambda}=\left(\Delta^{\prime}, f t^{\prime}\right)$, where $(\Delta, t)_{\Phi_{l}}^{\Lambda}=\left(\Delta^{\prime}, t^{\prime}\right)$. By induction hypothesis, $\left(\varnothing, t^{\prime} \sigma^{\prime}\right)=(\varnothing, \sigma(t))_{\Phi_{l}}^{\Lambda}$, and $\vdash \Delta^{\prime} \sigma^{\prime}$. The result follows, since $\sigma(f t)=f \sigma(t)$.

(tuple): If $s=\left(s_{1}, \ldots, s_{n}\right)$, then $\left(\Delta,\left(s_{1}, \ldots, s_{n}\right)\right)_{\Phi_{l}}^{\Lambda}=\left(\Delta^{\prime},\left(s_{1}^{\prime}, \ldots, s_{n}^{\prime}\right)\right)$, where $\left(\Delta, s_{i}\right)_{\Phi_{l}}^{\Lambda}=$ $\left(\Delta_{i}^{\prime}, s_{i}^{\prime}\right)$ and $\Delta^{\prime}=\Delta_{1}^{\prime} \cup \cdots \cup \Delta_{n}^{\prime}$. By induction, $\left(\varnothing, \sigma\left(s_{i}\right)\right)_{\Phi_{l}}^{\Lambda}=s_{i} \sigma^{\prime}$, and $\vdash \Delta_{i}^{\prime} \sigma^{\prime}$ for $1 \leq i \leq n$. Therefore $\vdash \Delta^{\prime} \sigma^{\prime}$, and the result follows since $\left(s_{1}^{\prime}, \ldots, s_{n}^{\prime}\right) \sigma^{\prime}=\left(s_{1}^{\prime} \sigma^{\prime}, \ldots, s_{n}^{\prime} \sigma^{\prime}\right)$.

The proof for function Right is similar to Left because of the syntax-directed nature of the translation. However, there is a difference in the case of a meta-application $Z_{i}^{n} t$, because in the right-hand side of a CRS rule the list of arguments $t$ may contain any kind of CRS metaterm, not just variables. The translation function Right deals with non-variable arguments by introducing explicit substitutions for atoms. Unlike Lemma 6.21, the instantiation lemma for Right relies on the use of the explicit substitution rules (see Definition 6.9)). We first state the correctness property for the rules in Definition 6.9, namely that the rules indeed specify the non-capturing atom-substitution operation.

Definition 6.22 ( $\sigma$-normal form of a term-in-context). We denote by $n f_{\sigma}(\Delta \vdash t)$ the normal form of the term-in-context $\Delta \vdash t$ with respect to the rules in Definition 6.9. It is uniquely defined modulo $\alpha$-equivalence [16].

Lemma 6.23 (Correctness of explicit substitution rules). Let $t, s$ be CRS terms (and therefore also nominal terms). Then $n f_{\sigma}(t[a \mapsto s]) \approx_{\alpha} t\{a \mapsto s\}$, where in the right-hand side $t\{a \mapsto s\}$ denotes the term obtained by substituting (using the capture-avoiding substitution of the CRS) a by $s$ in $t$.

Proof. The proof by induction is omitted since it is standard in explicit substitution calculi.

We have already proved in Lemma 6.14 that Right translates closed CRS meta-terms to closed NRS terms, and thanks to the safety conditions (see Definition 2.25) and Barendregt's convention (see Remark 2.24), bound variable names are all different, and also different from free variables, both in meta-terms and valuations. This ensures that, in the nominal translation, the explicit substitutions for atoms preserve the semantics of terms.

Lemma 6.24 (Right instantiation). Let $l \Rightarrow r$ be a CRS rule and $\Phi_{l}$ the function given in Definition 6.1 applied to l. Assume Right $(r)=\Delta \vdash r^{\prime}$, where according to Definition $6.10 \Delta=\left\{a_{k} \# Z_{i}^{n} \mid a_{k}\right.$ occurs bound above $Z_{i}^{n}$ in $\left.r\right\}$. Let $\sigma$ be a safe valuation such that $\operatorname{dom}(\sigma) \subseteq M V(l)$ (hence, $\sigma(r)$ is a CRS term).

Suppose $s^{\prime}=\llbracket s \rrbracket_{\Phi_{l}}$ is a recursive call in the translation of Right $(r)$, for any subterm $s$ of $r(e . g ., s=r)$, and $\sigma^{\prime}=\langle\sigma\rangle_{\phi_{l}}$ by Definition 6.19.

Then, $\llbracket \sigma(s) \rrbracket_{\Phi_{\varnothing}} \approx_{\alpha} n f_{\sigma}\left(s^{\prime} \sigma^{\prime}\right)$ and $\sigma^{\prime}$ satisfies $\Delta$, i.e., $\vdash \Delta \sigma^{\prime}$. 
Proof. By induction on the structure of $s$, using the fact that $\Delta \vdash r^{\prime}$ is closed by Lemma6.14. the syntactic equivalence between CRS terms and ground nominal terms (Remark 6.15) and the safety conditions given in Definition 2.25 and Remark 2.24.

Interesting cases to consider are $s=\left(s_{1}, \ldots, s_{n}\right)$ and also $s=Z_{i}^{n} t$.

- The case $s=\left(s_{1}, \ldots, s_{n}\right)$. By Definition 6.10 we have $\left(\llbracket\left(s_{1}, \ldots, s_{n}\right) \rrbracket_{\Phi_{l}}=\left(s_{1}^{\prime}, \ldots, s_{n}^{\prime}\right)\right.$ where $V\left(s_{1}^{\prime}, \ldots, s_{n}^{\prime}\right) \subseteq \operatorname{dom}\left(\sigma^{\prime}\right)$ and, by Remark 2.24, $A\left(i m g\left(\sigma^{\prime}\right)\right) \cap A\left(s_{1}^{\prime}, \ldots, s_{n}^{\prime}\right)=\varnothing$ such that $\left.\vdash \Delta \sigma^{\prime}\right|_{V\left(s_{1}^{\prime}, \ldots, s_{n}^{\prime}\right)}$ follows by induction hypothesis.

Now,

$$
\left(s_{1}^{\prime}, \ldots, s_{n}^{\prime}\right) \sigma^{\prime}=\left(s_{1}^{\prime} \sigma^{\prime}, \ldots, s_{n}^{\prime} \sigma^{\prime}\right) \quad \text { and } \quad \llbracket \sigma\left(s_{1}, \ldots, s_{n}\right) \rrbracket_{\Phi_{\varnothing}}=\left(\llbracket \sigma\left(s_{1}\right) \rrbracket_{\Phi_{\varnothing}}, \ldots, \llbracket \sigma\left(s_{n}\right) \rrbracket_{\Phi_{\varnothing}}\right)
$$

such that

$$
\llbracket \sigma\left(s_{1}\right) \rrbracket_{\Phi_{\varnothing}} \approx_{\alpha} n f_{\sigma}\left(s_{1}^{\prime} \sigma^{\prime}\right), \ldots, \llbracket \sigma\left(s_{n}\right) \rrbracket_{\Phi_{\varnothing}} \approx_{\alpha} n f_{\sigma}\left(s_{n}^{\prime} \sigma^{\prime}\right)
$$

by induction hypothesis.

- The case $\left(s=Z_{i}^{n} t\right)$. For the case where $t=()$, the result follows similarly to case (1) of (Mvar) in Lemma 6.21

For the case where $t=\left(t_{1}, \ldots, t_{n}\right)$, there is $\Phi_{l}\left(Z_{i}^{n}\right)=\left[a_{1}, \ldots, a_{n}\right]$ by Definition 6.1 where $\left[a_{1}, \ldots, a_{n}\right]$ is a list of distinct atoms by Remark 6.15 and then

$$
\llbracket Z_{i}^{n}\left(t_{1}, \ldots, t_{n}\right) \rrbracket_{\Phi_{l}}=\left(\left(a_{j 1} \llbracket t_{j 1} \rrbracket_{\Phi_{l}}\right) \cdots\left(a_{j k} \llbracket t_{j k} \rrbracket_{\Phi_{l}}\right) \cdot Z_{i}\right)\left[a_{m 1} \mapsto \llbracket t_{m 1} \rrbracket_{\Phi_{l}}\right] \ldots\left[a_{m k} \mapsto \llbracket t_{m k} \rrbracket \Phi_{l}\right],
$$

where $j 1, \ldots, j k, m 1, \ldots, m k \in\{1 \ldots n\}$ and $\llbracket t_{j 1} \rrbracket_{\Phi_{l}}, \ldots, \llbracket t_{j k} \rrbracket_{\Phi_{l}} \in \mathcal{A}$ by Definition 6.10 .

Assume, without loss of generality, $\sigma\left(Z_{i}^{n}\right)=\underline{\lambda}\left(b_{1} \ldots b_{n}\right) . s_{i}$. Following Definition 6.19 for $\langle\sigma\rangle_{\Phi_{l}}, \sigma^{\prime}\left(Z_{i}\right)=\left(a_{1} b_{1}\right) \cdots\left(a_{n} b_{n}\right) \cdot s_{i}$ where $s_{i}$ is a NRS ground term by Remark 6.15 where $\left\{a_{1}, \ldots, a_{n}\right\} \notin A\left(s_{i}\right)$ by Remark 2.24. Moreover, following the same remark we have $V(\sigma) \cap V(r)=\varnothing$, therefore it is the case that $\left.\sigma^{\prime}\right|_{Z_{i}}$ satisfies $\Delta,\left.\vdash \Delta \sigma^{\prime}\right|_{Z_{i}}$.

Hence, by application of explicit rules in Definition [6.9, $n f_{\sigma}\left(s^{\prime} \sigma^{\prime}\right) \approx_{\alpha}$ $s_{i}\left\{b_{1} \mapsto \llbracket t_{1} \rrbracket \Phi_{l} \sigma^{\prime}\right\} \cdots\left\{b_{n} \mapsto \llbracket t_{n} \rrbracket \Phi_{l} \sigma^{\prime}\right\}$ since $n f_{\sigma}(\cdot)$ correctly computes the substitution as stated in Lemma 6.23.

Now, $\llbracket \sigma\left(Z_{i}^{n}\left(t_{1}, \ldots, t_{n}\right)\right) \rrbracket_{\Phi_{\varnothing}}=s_{i}\left\{b_{1} \mapsto \llbracket \sigma\left(t_{1}\right) \rrbracket_{\Phi_{l}}\right\} \cdots\left\{b_{n} \mapsto \llbracket \sigma\left(t_{n}\right) \rrbracket_{\Phi_{l}}\right\}$, and the result follows by induction hypothesis.

The rest of the cases are omitted.

Let us denote by $\mathcal{R}$ the nominal rewriting system obtained by translating all the rules of the CRS $R$ according to Definition 6.16 and adding the rules for explicit substitution in Definition 6.9.

We are now ready to prove the correctness of the translated reduction relation.

Theorem 6.25 (Translation of CRS Rewrite Steps). Let $R=l \Rightarrow r$ be a CRS rule. Let $u$ be a CRS term.

If $u \Rightarrow_{R} v$ then $\vdash u \underset{\mathcal{R} \cup \sigma}{\stackrel{+}{\longrightarrow}} v$ using $\mathcal{R}=\mathcal{C}^{\mathcal{R}}(l, r)$ and the explicit substitution rules.

Proof. By Definition 6.16, $\mathcal{R}=\mathcal{C}^{\mathcal{R}}(l, r)=\nabla_{l} \cup \nabla_{r} \vdash l^{\prime} \rightarrow r^{\prime}$. It is a closed NRS rule by Lemma 6.18. Since $u, v$ are terms in CRS $R$, they are also ground terms in NRS $\mathcal{R}$ by Remark 6.15, and without loss of generality we can assume that $\nabla_{l} \cup \nabla_{r} \vdash l^{\prime} \rightarrow r^{\prime}$ does not mention any atom in $u$ (i.e., it is already freshened for $u$ ). If $u \Rightarrow_{R} v$ then there exists $p, \sigma$ such that $\left.u\right|_{p}=\sigma(l)$ where $p$ is a position in $u, \sigma$ a CRS valuation where $M V(l)=\operatorname{dom}(\sigma)$ and $v=u[\sigma(r)]_{p}$. Let $\sigma^{\prime}=\langle\sigma\rangle_{\Phi_{l}}$ according to Definition 6.19. By Lemma 6.21, if $\operatorname{Left}(l)=\left(\nabla_{l}, l^{\prime}\right)$ then $\operatorname{Left}(l \sigma)=\left(\varnothing, l^{\prime} \sigma^{\prime}\right)$ where $\sigma^{\prime}$ satisfies $\nabla_{l}$. Then, it is 
also the case that $\left.\vdash u\right|_{p} \approx_{\alpha} l^{\prime} \sigma^{\prime}$ by Remark 6.15. Moreover, $\sigma^{\prime}$ also satisfies $\nabla_{r}$ since we are using Barendregt convention.

It remains to prove that $u\left[r^{\prime} \sigma^{\prime}\right]_{p} \rightarrow^{*} v$. But this follows from Lemma 6.24, since $\operatorname{Right}(\sigma(r))=\left(\varnothing, n f_{\sigma}\left(r^{\prime} \sigma^{\prime}\right)\right)$ where $\operatorname{Right}(r)=\left(\nabla_{r}, r^{\prime}\right)$. Hence, $u \Rightarrow_{R} v$ with $v=u[\sigma(r)]_{p}$ implies $\vdash u \underset{\mathcal{R} \cup \sigma}{\stackrel{+}{\longrightarrow}} v$ where $v=u\left[n f_{\sigma}\left(r^{\prime} \sigma^{\prime}\right)\right]_{p}$.

We have designed an algorithm that correctly transforms CRS rules inot NRS closed rules. It improves over the function defined in [16] in two ways: we have fixed a bug in the translation of meta-variables in the scope of abstractions, and by using closed rewriting (see Definition 2.15) we are able to simulate the variable naming convention without adding extra freshness constraints, as shown in Example 6.17.

Next, we provide examples where both transformations are applied (NRSs to CRSs and back).

\section{EXAMPLES}

After describing the tools required to translate NRSs to CRSs (section 4) and back to NRSs (section 6), in this section we give two examples, which have been translated using the implementation available from [10].

7.1. Prenex Normal Form. We present here a translation back to NRSs by application of Definition 6.16 to the CRS rules obtained in Example 4.4. Beforehand we have applied the usual naming convention in rules (renaming bound variable $a$ to $b$ on the right-hand side). The resulting NRS is:

$$
\begin{array}{rll}
b \# P, b \# Q & \vdash \text { and }(P, \text { forall }([a] Q)) & \rightarrow \text { forall }([b] \operatorname{and}(P,(a b) \cdot Q)) \\
b \# P, b \# Q & \vdash \text { and }(\text { forall }([a] Q), P) & \rightarrow \text { forall }([b] \operatorname{and}((a b) \cdot Q, P)) \\
b \# P, b \# Q & \vdash \text { or }(P, \text { forall }([a] Q)) & \rightarrow \text { forall }([b] \operatorname{or}(P,(a b) \cdot Q)) \\
b \# P, b \# Q & \vdash \text { or }(\text { forall }([a] Q), P) & \rightarrow \text { forall }([b] \operatorname{or}((a b) \cdot Q, P)) \\
b \# P, b \# Q & \vdash \text { and }(P, \operatorname{exist~}([a] Q)) & \rightarrow \text { exists }([b] \operatorname{and}(P,(a b) \cdot Q)) \\
b \# P, b \# Q & \vdash \text { and }(\operatorname{exists}([a] Q), P) & \rightarrow \operatorname{exists}([b] \operatorname{and}((a b) \cdot Q, P)) \\
b \# P, b \# Q & \vdash \operatorname{or}(P, \operatorname{exists}([a] Q) & \rightarrow \operatorname{exists}([b] \operatorname{or}(P,(a b) \cdot Q)) \\
b \# P, b \# Q & \vdash \operatorname{or}(\operatorname{exists}([a] Q), P) & \rightarrow \operatorname{exists}[b] \operatorname{or}((a b) \cdot Q, P) \\
b \# Q & \vdash \operatorname{not}(\operatorname{exists}([a] Q)) & \rightarrow \operatorname{forall}([b] \operatorname{not}((a b) \cdot Q)) \\
b \# Q & \vdash \operatorname{not}(\operatorname{forall}([a] Q)) & \rightarrow \operatorname{exists}([b] \operatorname{not}((a b) \cdot Q)) .
\end{array}
$$

Notice that a matching $\sigma=[Q \mapsto a]$ with the left-hand side of a rule leads to the expected result when applied to the right-hand side of the rule thanks to the swapping $(a b)$. Also, the freshness condition $a \# P$ in the initial set of NRS rules (see Example 2.12) is shown here as $b \# P$ because of the variable convention applied beforehand, $b \# Q$ is added to the set of freshness conditions (so, the rules remain closed). This does not alter the semantics, as we can see by translating back. When we translate back to CRS the first NRS rule

$$
b \# P, b \# Q \vdash \text { and }(P, \text { forall }([a] Q)) \rightarrow \text { forall }([b] \text { and }(P,(a b) \cdot Q))
$$

using Definition 4.2, we obtain the CRS rule

$$
\text { and }(P, \text { forall }([a] Q(a))) \rightarrow \text { forall }([b] \text { and }(P, Q(b)))
$$

as expected. 
7.2. Simulating $\beta$-reduction. Consider the $\left(\beta_{\mathrm{lam}}\right)$ rule given in Example 4.5. First, we apply Barendregt's convention to the CRS rule so that each bound variable is distinct, obtaining:

$$
\operatorname{app}(\operatorname{lam}([a] \operatorname{lam}([b] X(a, b))), Y) \Rightarrow \operatorname{lam}([d] \operatorname{app}(\operatorname{lam}([c] X(c, d)), Y))
$$

Its translation to NRSs is the following:

$$
d \# Y, d \# X, c \# X \vdash \operatorname{app}(\operatorname{lam}[a] \operatorname{lam}[b] X, Y) \rightarrow \operatorname{lam}[d] \operatorname{app}(\operatorname{lam}[c](a c)(b d) \cdot X, Y)
$$

And when translated back to CRSs by means of Definition 4.2, we obtain the same CRS rule as expected.

\section{Conclusions and Future Work}

We have shown two extensions of first-order rewriting, CRSs and NRSs, to be closely related. The main differences are in the meta-language used, NRSs do not rely on the $\lambda$-calculus, employing instead permutations of atoms and a freshness relation to axiomatise $\alpha$-equivalence. Also NRS rules are more general than CRS rules in that unabstracted atoms may occur in rules. On the other hand, CRSs rely on the $\lambda$-calculus to generate its rewrite relation and CRS rules are closed by definition.

We have shown that despite these differences it is possible to translate between these formalisms, under certain conditions. We have given a translation function which transforms the class of closed nominal rewriting systems into CRS systems. We have shown some nontrivial examples to support our work; see [10, 9] for a Haskell implementation.

Moreover, existing translation algorithms between CRSs and HRSs [36], $\rho$-calculus [3] and ERSs [20] allow transformations from NRSs to any of these systems and vice versa.

Although previous translations between nominal and higher-order syntax exist [27, 6], our work differs from these by focusing on a syntax-directed translation of terms and rewrite rules that preserves the rewriting relation, which is key to the translation of properties such as confluence and termination. We have also corrected and improved a previous algorithm translating CRSs to NRSs originally found in [16].

Since nominal terms have good algorithmic properties, we could translate CRSs to NRSs in order to take advantage of existing nominal procedures (i.e., orderings, completion) and then transfer back the results. The extension to typed systems (adapting the type systems developed for NRSs [12, 13] to CRSs) is also left for future work.

Acknowledgements. We thank Elliot Fairweather and Christian Urban for many helpful discussions, and Jamie Gabbay for providing the macro for $И$. We also thank the anonymous referees for their valuable comments.

\section{REFERENCES}

[1] F. Baader and T. Nipkow. Term rewriting and all that. Cambridge University Press, 1988.

[2] H. P. Barendregt. The lambda calculus, its syntax and semantics, volume 103 of Studies in Logic and the Foundation of Mathematics. North-Holland, revised edition, 1984.

[3] C. Bertolissi, H. Cirstea, and C. Kirchner. Expressing combinatory reduction systems derivations in the rewriting calculus. Higher-Order and Symbolic Computation, 19:00110869, 2006.

[4] M. Bezem, J. W. Klop, and R. de Vrijer, editors. Term rewrite systems by TeReSe, volume 55 of Cambridge Tracts in Theoretical Computer Science. Cambridge University Press, 2003. 
[5] C. Calvès and M. Fernández. Matching and alpha-equivalence check for nominal terms. Journal of Computer and System Sciences, 2009. Special issue: Selected papers from WOLLIC 2008.

[6] J. Cheney. Relating nominal and higher-order pattern unification. In Proceedings of International Workshop in Unification, pages 104-119, 2005.

[7] H. Cirstea and C. Kirchner. The rewriting calculus - Part I. Logic Journal of the Interest Group in Pure and Applied Logics, 9(3):427-463, May 2001.

[8] H. Cirstea and C. Kirchner. The rewriting calculus - Part II. IGPL, 9(3):377-410, 2001.

[9] J. Domínguez. A tool to apply nominal recursive path ordering to nominal rules. 2014. Available from http://www.inf.kcl.ac.uk/pg/domijesu/nrpo.tgz.

[10] J. Domínguez. A tool to translate between closed NRSs and CRSs. 2014. Available from http://www.inf.kcl.ac.uk/pg/domijesu/NRS2CRS.tar.gz.

[11] J. Domínguez and M. Fernández. Relating nominal and higher-order rewriting. In Mathematical Foundations of Computer Science 2014 - 39th International Symposium, Budapest, Hungary, August 25-29, 2014. Proceedings, Part I, pages 244-255, 2014.

[12] E. Fairweather, M. Fernández, and M. J. Gabbay. Principal types for nominal theories. Lecture Notes in Computer Science, 6914 LNCS:160-172, 2011.

[13] M. Fernández and M. J. Gabbay. Curry-style types for nominal terms. In T. Altenkirch and C. McBride, editors, Types for Proofs and Programs, volume 4502 of Lecture Notes in Computer Science, pages 125139. Springer Berlin Heidelberg, 2007.

[14] M. Fernández and M. J. Gabbay. Nominal rewriting. Information and Computation, 205(6):917-965, 2007.

[15] M. Fernández and M. J. Gabbay. Closed nominal rewriting and efficiently computable nominal algebra equality. In Proceedings of International Workshop on Logical Frameworks and Meta-Languages: Theory and Practice, pages 37-51, 2010.

[16] M. Fernández, M. J. Gabbay, and I. Mackie. Nominal rewriting systems. In Proceedings of the 6th ACM SIGPLAN international conference on Principles and practice of declarative programming, PPDP '04, pages 108-119, New York, NY, USA, 2004. ACM.

[17] M. Fernández and A. Rubio. Nominal completion for rewrite systems with binders. In A. Czumaj, K. Mehlhorn, A. Pitts, and R. Wattenhofer, editors, Automata, Languages, and Programming, volume 7392 of Lecture Notes in Computer Science, pages 201-213. Springer Berlin Heidelberg, 2012.

[18] M. J. Gabbay and A. Mathijssen. Capture-avoiding substitution as a nominal algebra. Formal Aspects of Computation, 20(4-5):451-479, 2008.

[19] M. J. Gabbay and A. M. Pitts. A new approach to abstract syntax with variable binding. Formal Aspects of Computing, 13(3-5):341-363, 2002.

[20] J. Glauert, D. Kesner, and Z. Khasidashvili. Expression reduction systems and extensions: An overview. In A. Middeldorp, V. van Oostrom, F. van Raamsdonk, and R. Vrijer, editors, Processes, Terms and Cycles: Steps on the Road to Infinity, volume 3838 of Lecture Notes in Computer Science, pages 496-553. Springer Berlin Heidelberg, 2005.

[21] M. Hamana. Semantic labelling for proving termination of combinatory reduction systems. In S. Escobar, editor, Functional and Constraint Logic Programming, volume 5979 of Lecture Notes in Computer Science, pages 62-78. Springer Berlin Heidelberg, 2010.

[22] J.-P. Jouannaud. Higher-order rewriting: Framework, confluence and termination. In A. Middeldorp, V. van Oostrom, F. van Raamsdonk, and R. Vrijer, editors, Processes, Terms and Cycles: Steps on the Road to Infinity, volume 3838 of Lecture Notes in Computer Science, pages 224-250. Springer Berlin Heidelberg, 2005.

[23] Z. Khasidashvili. Expression reduction systems. In Proc. of I. Vekua Institute of Applied Mathematics, volume 36, pages 200-220, 1990 .

[24] J. W. Klop. Combinatory reduction systems. PhD thesis, Utrecht University, 1980.

[25] J. W. Klop, V. van Oostrom, and F. van Raamsdonk. Combinatory reduction systems: Introduction and survey. Theoretical Computer Science, 121:279-308, 1993.

[26] C. Kop. Simplifying algebraic functional systems. In F. Winkler, editor, Algebraic Informatics, volume 6742 of Lecture Notes in Computer Science, pages 201-215. Springer Berlin Heidelberg, 2011.

[27] J. Levy and M. Villaret. Nominal unification from a higher-order perspective. ACM Transactions in Computer Logic, 13(2):10:1-10:31, 2012. 
[28] R. Mayr and T. Nipkow. Higher-order rewrite systems and their confluence. Theoretical Computer Science, 192(1):3 - 29, 1998.

[29] T. Nipkow. Higher-order critical pairs. In Proceedings of IEEE Symposium on Logic in Computer Science, pages 342-349, 1991.

[30] T. Nipkow and C. Prehofer. Higher-order rewriting and equational reasoning. In W. Bibel and P. Schmitt, editors, Automated Deduction - A Basis for Applications. Volume I: Foundations, volume 8 of Journal of Applied Logic, pages 399-430. Kluwer, 1998.

[31] A. M. Pitts. Nominal logic, a first order theory of names and binding. Information and Computation, 186:165-193, 2003.

[32] A. M. Pitts. Nominal sets: names and symmetry in computer science, volume 57 of Cambridge Tracts in Theoretical Computer Science. Cambridge University Press, 2013.

[33] B. Sagan. The symmetric group, volume 203. Springer-Verlag New York, 2 edition, 2001.

[34] C. Urban, A. M. Pitts, and M. Gabbay. Nominal unification. In M. Baaz and J. Makowsky, editors, Computer Science Logic, volume 2803 of Lecture Notes in Computer Science, pages 513-527. Springer Berlin Heidelberg, 2003.

[35] C. Urban, A. M. Pitts, and M. J. Gabbay. Nominal unification. Theoretical Computer Science, 323(13):473 - 497, 2004.

[36] V. van Oostrom and F. van Raamsdonk. Comparing combinatory reduction systems and higher-order rewrite systems. In J. Heering, K. Meinke, B. Mller, and T. Nipkow, editors, Higher-Order Algebra, Logic, and Term Rewriting, volume 816 of Lecture Notes in Computer Science, pages 276-304. Springer Berlin Heidelberg, 1994.

[37] F. van Raamsdonk. Higher-order rewriting. In P. Narendran and M. Rusinowitch, editors, Rewriting Techniques and Applications, volume 1631 of Lecture Notes in Computer Science, pages 220-239. Springer Berlin Heidelberg, 1999. 